\title{
Second-Order Two-Sided Estimates in Nonlinear Elliptic Problems
}

\author{
Andrea Cianchi \& Vladimir G. MaZ'ya
}

Communicated by V. ŠvERÁK

\begin{abstract}
Best possible second-order regularity is established for solutions to $p$-Laplacian type equations with $p \in(1, \infty)$ and a square-integrable right-hand side. Our results provide a nonlinear counterpart of the classical $L^{2}$-coercivity theory for linear problems, which is missing in the existing literature. Both local and global estimates are obtained. The latter apply to solutions to either Dirichlet or Neumann boundary value problems. Minimal regularity on the boundary of the domain is required, although our conclusions are new even for smooth domains. If the domain is convex, no regularity of its boundary is needed at all.
\end{abstract}

\section{Introduction}

A prototypical result in the theory of elliptic equations asserts that, if $\Omega$ is a bounded open set in $\mathbb{R}^{n}, n \geqq 2$, with $\partial \Omega \in C^{2}$, and $u$ is the weak solution to the Dirichlet problem for the inhomegenous Laplace equation whose right-hand side $f \in L^{2}(\Omega)$, then $u \in W^{2,2}(\Omega)$. Moreover, a two-sided estimate for $\left\|\nabla^{2} u\right\|_{L^{2}(\Omega)}$ holds in terms of $\|f\|_{L^{2}(\Omega)}$. This can be traced back to [9] for $n=2$, and to [56] for $n \geqq 3$. A comprehensive analysis of related topics can be found in [2], [36, Chapter 10], [42, Chapter 3], [53, Chapter 14].

The purpose of the present paper is to offer an optimal natural analogue of this classical result for a class of quasilinear elliptic problems in divergence form. This class encompasses the inhomegenous $p$-Laplace equation

$$
-\operatorname{div}\left(|\nabla u|^{p-2} \nabla u\right)=f \quad \text { in } \Omega
$$

for any $p \in(1, \infty)$ and any right-hand side $f \in L^{2}(\Omega)$. For solutions $u$ to equation (1.1), coupled either with Dirichlet or Neumann homogeneous boundary conditions, our result tells us that

$$
|\nabla u|^{p-2} \nabla u \in W^{1,2}(\Omega) \text { if and only if } f \in L^{2}(\Omega) .
$$


Moreover, the norm of $|\nabla u|^{p-2} \nabla u$ in $W^{1,2}(\Omega)$ and the norm of $f$ in $L^{2}(\Omega)$ are equivalent. Obviously, the difficult implication in (1.2) amounts to showing that, if $f \in L^{2}(\Omega)$, then $|\nabla u|^{p-2} \nabla u \in W^{1,2}(\Omega)$.

A distinctive trait of the precise statement of (1.2) is the minimal regularity imposed on $\partial \Omega$. In particular, if $\Omega$ is convex, no additional assumption has to be required on $\partial \Omega$. However, we stress that the conclusions to be derived are new even for smooth domains. A counterpart of (1.2) for local solutions to equation (1.1) is establshed as well.

An additional significant feature of the results to be presented is that they also apply to a very weak notion of solutions. These solutions need not be even weakly differentiable, if $p$ is too close to 1 , yet satisfy (1.2), provided that $\nabla u$ is suitably interpreted.

As far as we know, no equivalence result in the spirit of (1.2) is available in the literature, although a regularity theory for nonlinear equations in divergence form, modeled upon the $p$-Laplacian, has extensively been developed in the last 50 years. This includes the classics [17,27,28,33,37,38,43,44,58,60-62], and the newer advances $[11,13,13,14,22,31,41]$. In particular, a standard result concerning second-order differentiability properties of $p$-harmonic functions, i.e. local solutions $u$ to equation (1.1) with $f=0$, asserts that the nonlinear expression of the gradient $|\nabla u|^{\frac{p-2}{2}} \nabla u$ belongs to $W_{\text {loc }}^{1,2}(\Omega)$ —see [61] for $p \in(2, \infty)$, and [17] for every $p \in(1, \infty)$. Second-order regularity for inhomogeneous equations has been the object of research in recent years-see e.g. $[8,15,24]$ in this connection. Results on the differentiability of the function $|\nabla u|^{p-2} \nabla u$ for solutions to equation (1.1) are available, but require additional regularity properties on the right-hand side $f$. Moreover, they either just deal with local solutions [4,46], or entail smoothness assumptions on $\partial \Omega$ [26]. Fractional-order regularity for the gradient of solutions to nonlinear equations of $p$-Laplacian type is the subject of $[4,16,54]$. An earlier contribution in this direction is [57].

To conclude this preliminary overview, let us point out that the equivalence principle (1.2) raises the natural problem of a more general result of the same nature for $f$ in $L^{q}(\Omega)$ with $q \neq 2$, or in other function spaces.

\section{Main Results}

Although our main focus is on global estimates for solutions to boundary value problems, we begin our discussion with a local bound for local solutions, which is of independent interest. The equations under consideration have the form

$$
-\operatorname{div}(a(|\nabla u|) \nabla u)=f \quad \text { in } \Omega,
$$

where $\Omega$ is any open set in $\mathbb{R}^{n}$, and $f \in L_{\text {loc }}^{2}(\Omega)$. The function $a:(0, \infty) \rightarrow(0, \infty)$ is of class $C^{1}(0, \infty)$, and such that

$$
-1<i_{a} \leqq s_{a}<\infty
$$

where

$$
i_{a}=\inf _{t>0} \frac{t a^{\prime}(t)}{a(t)} \quad \text { and } \quad s_{a}=\sup _{t>0} \frac{t a^{\prime}(t)}{a(t)},
$$


and $a^{\prime}$ stands for the derivative of $a$. Assumption (2.2) ensures that the differential operator in (2.1) satisfies ellipticity and monotonicity conditions, not necessarily of power type [21,22]. Regularity for equations governed by generalized nonlinearities of this kind has also been extensively studied-see e.g. [5, 12, 19, 20,29,30,39,44, $47,59]$. Observe that the standard $p$-Laplace operator corresponds to the choice $a(t)=t^{p-2}$, with $p>1$. Clearly, $i_{a}=s_{a}=p-2$ in this case.

As was already warned in Section 1 , due to the mere square summability assumption on the function $f$, solutions to equation (2.1) may have to be understood in a suitable generalized sense, even in the case of the $p$-Laplacian. We shall further comment on this at the end of this section. Precise definitions can be found in Sections 4 and 5.

In what follows, $B_{r}(x)$ denotes the ball with radius $r>0$, centered at $x \in \mathbb{R}^{n}$. The simplified notation $B_{r}$ is employed when information on the center is irrelevant. In this case, balls with different radii appearing in the same formula (or proof) will be tacitly assumed to have the same center.

Theorem 2.1. [Local estimate] Assume that the function $a \in C^{1}(0, \infty)$, and satisfies condition (2.2). Let $\Omega$ be any open set in $\mathbb{R}^{n}$, with $n \geqq 2$, and let $f \in L_{\text {loc }}^{2}(\Omega)$. Let $u$ be a generalized local solution to equation (2.1). Then

$$
a(|\nabla u|) \nabla u \in W_{\text {loc }}^{1,2}(\Omega),
$$

and there exists a constant $C=C\left(n, i_{a}, s_{a}\right)$ such that

$$
\|a(|\nabla u|) \nabla u\|_{W^{1,2}\left(B_{R}\right)} \leqq C\left(\|f\|_{L^{2}\left(B_{2 R}\right)}+\left(R^{-\frac{n}{2}}+R^{-\frac{n}{2}-1}\right)\|a(|\nabla u|) \nabla u\|_{L^{1}\left(B_{2 R}\right)}\right)
$$

for any ball $B_{2 R} \subset \subset \Omega$.

Remark 2.2. Observe that the expression $a(|\nabla u|) \nabla u$ agrees with $|\nabla u|^{p-2} \nabla u$ when the differential operator in equation (2.1) is the $p$-Laplacian, and hence differs in the exponent of $|\nabla u|$ from the classical results recalled above about $p$-harmonic functions.

Our global results concern Dirichlet or Neumann problems, with homogeneous boundary data, associated with equation (2.1); namely, Dirichlet problems of the form

$$
\begin{cases}-\operatorname{div}(a(|\nabla u|) \nabla u)=f & \text { in } \Omega \\ u=0 & \text { on } \partial \Omega\end{cases}
$$

and Neumann problems of the form

$$
\begin{cases}-\operatorname{div}(a(|\nabla u|) \nabla u)=f & \text { in } \Omega \\ \frac{\partial u}{\partial v}=0 & \text { on } \partial \Omega .\end{cases}
$$

Here, $\Omega$ is a bounded open set in $\mathbb{R}^{n}, v$ denotes the outward unit vector on $\partial \Omega$, $f \in L^{2}(\Omega)$, and $a:(0, \infty) \rightarrow(0, \infty)$ is as above. Of course, the compatibility condition

$$
\int_{\Omega} f \mathrm{~d} x=0
$$


has to be required when dealing with (2.7).

A basic version of the global second-order estimates for the solutions to (2.6) and (2.7) holds in any bounded convex open set $\Omega \subset \mathbb{R}^{n}$.

Theorem 2.3. [Global estimate in convex domains] Assume that the function $a \in C^{1}(0, \infty)$, and satisfies condition (2.2). Let $\Omega$ be any bounded convex open set in $\mathbb{R}^{n}$, with $n \geqq 2$, and let $f \in L^{2}(\Omega)$. Let $u$ be the generalized solution to either the Dirichlet problem (2.6), or the Neumann problem (2.7). Then

$$
a(|\nabla u|) \nabla u \in W^{1,2}(\Omega) .
$$

Moreover,

$$
C_{1}\|f\|_{L^{2}(\Omega)} \leqq\|a(|\nabla u|) \nabla u\|_{W^{1,2}(\Omega)} \leqq C_{2}\|f\|_{L^{2}(\Omega)}
$$

for some constants $C_{1}=C_{1}\left(n, s_{a}\right)$ and $C_{2}=C_{2}\left(\Omega, i_{a}, s_{a}\right)$.

Heuristically speaking, the validity of a global estimate in Theorem 2.3 is related to the fact that the second fundamental form on the boundary of a convex set is semidefinite. In the main result of this paper, the convexity assumption on $\Omega$ is abandoned. Dropping signature information on the (weak) second fundamental form on $\partial \Omega$ calls for an assumption on its summability. We assume that $\Omega$ is bounded and a Lipschitz domain. This means that, in a neighborhood of each boundary point, $\Omega$ agrees with the subgraph of a Lipschitz continuous function of $(n-1)$ variables. We also assume that this function is twice weakly differentiable, and that its second-order derivatives belong to either the weak Lebesgue space $L^{n-1}$, called $L^{n-1, \infty}$, or the weak Zygmund space $L \log L$, called $L^{1, \infty} \log L$, according to whether $n \geqq 3$ or $n=2$. This will be denoted by $\partial \Omega \in L^{n-1, \infty}$, and $\partial \Omega \in L^{1, \infty} \log L$, respectively. As a consequence, the weak second fundamental form $\mathcal{B}$ on $\partial \Omega$ belongs to the same weak type spaces with respect to the $(n-1)$ dimensional Hausdorff measure $\mathcal{H}^{n-1}$ on $\partial \Omega$. Our key summability assumption on $\mathcal{B}$ amounts to

$$
\lim _{r \rightarrow 0^{+}}\left(\sup _{x \in \partial \Omega}\|\mathcal{B}\|_{L^{n-1, \infty}\left(\partial \Omega \cap B_{r}(x)\right)}\right)<c \quad \text { if } n \geqq 3,
$$

or

$$
\lim _{r \rightarrow 0^{+}}\left(\sup _{x \in \partial \Omega}\|\mathcal{B}\|_{L^{1, \infty} \log L\left(\partial \Omega \cap B_{r}(x)\right)}\right)<c \quad \text { if } \quad n=2,
$$

for a suitable constant $c=c\left(L_{\Omega}, d_{\Omega}, n, i_{a}, s_{a}\right)$. Here, $L_{\Omega}$ denotes the Lipschitz constant of $\Omega$, and $d_{\Omega}$ its diameter. Let us emphasize that such an assumption is essentially sharp—see Remark 2.5 below.

Theorem 2.4. [Global estimate in minimally regular domains] Assume that the function a $\in C^{1}(0, \infty)$, and satisfies condition (2.2). Let $\Omega$ be a bounded Lipschitz domain in $\mathbb{R}^{n}, n \geqq 2$ such that $\partial \Omega \in W^{2} L^{n-1, \infty}$ if $n \geqq 3$, or $\partial \Omega \in W^{2} L^{1, \infty} \log L$ if $n=2$. Assume that $f \in L^{2}(\Omega)$, and let $u$ be the generalized solution to either the Dirichlet problem (2.6), or the Neumann problem (2.7). There exists a constant $c=c\left(L_{\Omega}, d_{\Omega}, n, i_{a}, s_{a}\right)$ such that, if $\Omega$ fulfils (2.11) or (2.12) for such a constant $c$, then

$$
a(|\nabla u|) \nabla u \in W^{1,2}(\Omega) .
$$


Moreover,

$$
C_{1}\|f\|_{L^{2}(\Omega)} \leqq\|a(|\nabla u|) \nabla u\|_{W^{1,2}(\Omega)} \leqq C_{2}\|f\|_{L^{2}(\Omega)}
$$

for some positive constants $C_{1}=C_{1}\left(n, s_{a}\right)$ and $C_{2}=C_{2}\left(\Omega, i_{a}, s_{a}\right)$.

We conclude this section with some remarks on Theorems 2.1, 2.3 and 2.4.

Remark 2.5. Assumption (2.11), or (2.12), cannot be weakened in Theorem 2.4 for all equations of the form appearing in (2.6) and (2.7). This can be shown, for instance, when $n=3$ and $a(t)=t^{p-2}$, with $p \in\left(\frac{3}{2}, 2\right]$. Indeed, in [40] open sets $\Omega \subset \mathbb{R}^{3}$, with $\partial \Omega \in W^{2} L^{2, \infty}$ (but whose limit in (2.11) is not small enough), are exhibited where the solution $u$ to problem (2.6), with a smooth $f$, is such that $|\nabla u|^{p-2} \nabla u \notin W^{1,2}(\Omega)$. Moreover, if $a$ is constant, there exist open sets $\Omega \subset \mathbb{R}^{2}$, with $\partial \Omega \in W^{2} L^{1, \infty} \log L$, for which the limit in (2.12) exceeds some explicit threshold, and where the solution $u$ to problem (2.6) fails to belong to $W^{2,2}(\Omega)$ [50] (see also [53, Section 14.6.1]).

Remark 2.6. Condition (2.11) is certainly fulfilled if $\partial \Omega \in W^{2, n-1}$, and (2.12) is fulfilled if $\partial \Omega \in W^{2} L \log L$, or, a fortiori, if $\partial \Omega \in W^{2, q}$ for some $q>1$. This follows from the embedding of $L^{n-1}$ into $L^{n-1, \infty}$ and of $L \log L$ (or $L^{q}$ ) into $L^{1, \infty} \log L$ for $q>1$, and from the absolute continuity of the norm in any Lebesgue and Zygmund space. Notice also that, since the Lorentz space $L^{n-1,1} \varsubsetneqq L^{n-1}$, assumption (2.11) is, in particular, weaker than requiring that $\partial \Omega \in W^{2} L^{n-1,1}$. The latter condition has been shown to ensure the global boundedness of the gradient of the solutions to problems (2.6) or (2.7), for $n \geqq 3$, provided that $f$ belongs to the Lorentz space $L^{n, 1}(\Omega)[21,22]$. Note that hypothesis $(2.11)$ does not imply that $\partial \Omega \in C^{1,0}$, a property that is instead certainly fulfilled under the stronger condition that $\partial \Omega \in W^{2} L^{n-1,1}$.

Remark 2.7. The global gradient bound mentioned in Remark 2.6 enables one to show, via a minor variant in the proof of Theorems $2.3-2.4$, that the solutions to problems (2.6) and (2.7) are actually in $W^{2,2}(\Omega)$, provided that

$$
\inf _{t \in[0, M]} a(t)>0
$$

for every $M>0$, and $f$ and $\Omega$ have the required regularity for the relevant gradient bound to hold. A parallel result holds for local solutions to the equation (2.1), thanks to a local gradient estimate from [5], extending [31]. To be more specific, if $f \in L_{\text {loc }}^{n, 1}(\Omega)$, and $u$ is a generalized local solution to equation (2.1), then

$$
u \in W_{\mathrm{loc}}^{2,2}(\Omega) .
$$

Moreover, if $n \geqq 3, f \in L^{n, 1}(\Omega), \partial \Omega \in W^{2} L^{n-1,1}$, and $u$ is the generalized solution to either the Dirichlet problem (2.6), or the Neumann problem (2.7), then

$$
u \in W^{2,2}(\Omega) .
$$

Equation (2.17) continues to hold if $\Omega$ is any bounded convex domain in $\mathbb{R}^{n}$, whatever $\partial \Omega$ is. 
Let us stress that these conclusion may fail if assumption (2.15) is dropped. This can be verified, for instance, on choosing $a(t)=t^{p-2}$, i.e. the $p$-Laplace operator, and considering functions of the form $u(x)=\left|x_{1}\right|^{\beta}$, where $x=\left(x_{1}, \ldots, x_{n}\right)$ and $\beta>1$. These functions are local solutions to (2.1) with $f \in L_{\mathrm{loc}}^{n, 1}\left(\mathbb{R}^{n}\right)$ (and even $\left.f \in L_{\mathrm{loc}}^{\infty}\left(\mathbb{R}^{n}\right)\right)$ provided that $p$ is large enough, but $u \notin W_{\mathrm{loc}}^{2,2}\left(\mathbb{R}^{n}\right)$ if $\beta \leqq \frac{3}{2}$. In fact, $u \notin W_{\text {loc }}^{2, q}\left(\mathbb{R}^{n}\right)$ for any given $q>1$, if $\beta$ is sufficiently close to 1 .

Remark 2.8. Weak solutions to problems (2.6) or (2.7), namely distributional solutions belonging to the energy space associated with the relevant differential operator, need not exist if $f$ is merely in $L^{2}(\Omega)$. It is well-known that this phenomenon occurs in the model case of the $p$-Laplace equation, if $p$ is not large enough for $L^{2}(\Omega)$ to be contained in the dual of $W^{1, p}(\Omega)$. However, weaker definitions of solutions to boundary value problems for this equation, ensuring their uniqueness, which apply to any $p \in(1, \infty)$ and even to right-hand sides $f \in L^{1}(\Omega)$, are available in the literature $[3,6,10,25,31,45,51,55]$. Among the diverse, but a posteriori equivalent, definitions, we shall adopt that (adjusted to the framework under consideration in this paper) of a solution which is the limit of a sequence of solutions to problems whose right-hand sides are smooth and converge to $f$ [25]. This will be called a generalized solution throughout. A parallel notion of generalized local solution to (2.1) will be empolyed. A generalized solution need not be weakly differentiable. However, it is associated with a vector-valued function on $\Omega$, which plays the role of a substitute for its gradient in the distributional definition of solution. With some abuse of notation, this is the meaning attributed to $\nabla u$ in the statements of Theorems 2.1, 2.3 and 2.4.

A definition of generalized solution to problem (2.6) and to problem (2.7) is given in Section 4, where an existence, uniquess and first-order summability result from [23] is also recalled. Note that, owing to its uniqueness, this kind of generalized solution agrees with the weak solution whenever $f$ is summable enough, depending on the nonlinearity of the differential operator, for a weak solution to exist. Generalized local solutions to equation (2.1) are defined in Section 5.

\section{A Differential Inequality}

The subject of this section is a lower bound for the square of the differential operator on the left-hand side of the equations in (2.6) and (2.7) in terms of an operator in divergence form, plus (a positive constant times) the gradient of $a(|\nabla u|) \nabla u$ squared. This is a critical step in the proof of our main results, and is the content of the following lemma:

Lemma 3.1. Let $a:[0, \infty) \rightarrow[0, \infty)$ be a function of the form $a(t)=g\left(t^{2}\right)$ for some function $g \in C^{1}[0, \infty)$, and such that $a(t)>0$ if $t>0$. Assume that the first inequality in (2.2) holds. Then there exists a positive constant $C=C\left(n, i_{a}\right)$ such that 


$$
\begin{aligned}
(\operatorname{div}(a(|\nabla u|) \nabla u))^{2} \geqq & \sum_{j=1}^{n}\left(a(|\nabla u|)^{2} u_{x_{j}} \Delta u\right)_{x_{j}} \\
& -\sum_{i=1}^{n}\left(a(|\nabla u|)^{2} \sum_{j=1}^{n} u_{x_{j}} u_{x_{i} x_{j}}\right)_{x_{i}}+C a(|\nabla u|)^{2}\left|\nabla^{2} u\right|^{2}
\end{aligned}
$$

for every function $u \in C^{3}(\Omega)$. Here, $\left|\nabla^{2} u\right|=\left(\sum_{i, j=1}^{n} u_{x_{i} x_{j}}^{2}\right)^{\frac{1}{2}}$.

Proof. Let $u \in C^{3}(\Omega)$. Computations show that

$$
\begin{aligned}
(\operatorname{div} & (a(|\nabla u|) \nabla u))^{2}=\left(a(|\nabla u|) \Delta u+a^{\prime}(|\nabla u|) \nabla|\nabla u| \cdot \nabla u\right)^{2} \\
= & a(|\nabla u|)^{2}\left((\Delta u)^{2}-\left|\nabla^{2} u\right|^{2}\right)+a(|\nabla u|)^{2}\left|\nabla^{2} u\right|^{2}+ \\
& +a^{\prime}(|\nabla u|)^{2}(\nabla|\nabla u| \cdot \nabla u)^{2}+2 a(|\nabla u|) a^{\prime}(|\nabla u|) \Delta u \nabla|\nabla u| \cdot \nabla u \\
= & a(|\nabla u|)^{2}\left(\sum_{j=1}^{n}\left(u_{x_{j}} \Delta u\right)_{x_{j}}-\sum_{i, j=1}^{n}\left(u_{x_{j}} u_{x_{i} x_{j}}\right)_{x_{i}}\right)+a(|\nabla u|)^{2}\left|\nabla^{2} u\right|^{2} \\
& +a^{\prime}(|\nabla u|)^{2}(\nabla|\nabla u| \cdot \nabla u)^{2}+2 a(|\nabla u|) a^{\prime}(|\nabla u|) \Delta u \nabla|\nabla u| \cdot \nabla u \\
= & \sum_{j=1}^{n}\left(a(|\nabla u|)^{2} u_{x_{j}} \Delta u\right)_{x_{j}}-\sum_{i, j=1}^{n}\left(a(|\nabla u|)^{2} u_{x_{j}} u_{x_{i} x_{j}}\right)_{x_{i}} \\
& -2 a(|\nabla u|) a^{\prime}(|\nabla u|)\left(\Delta u \nabla|\nabla u| \cdot \nabla u-\sum_{i, j=1}^{n}|\nabla u|_{x_{i}} u_{x_{j}} u_{x_{i} x_{j}}\right) \\
& +a(|\nabla u|)^{2}\left|\nabla^{2} u\right|^{2}+a^{\prime}(|\nabla u|)^{2}(\nabla|\nabla u| \cdot \nabla u)^{2} \\
& +2 a(|\nabla u|) a^{\prime}(|\nabla u|) \Delta u \nabla|\nabla u| \cdot \nabla u \\
= & \sum_{j=1}^{n}\left(a(|\nabla u|)^{2} u_{x_{j}} \Delta u\right)_{x_{j}}-\sum_{i, j=1}^{n}\left(a(|\nabla u|)^{2} u_{x_{j}} u_{x_{i} x_{j}}\right)_{x_{i}} \\
& +2 a(|\nabla u|) a^{\prime}(|\nabla u|) \sum_{i, j=1}^{n}|\nabla u|_{x_{i}} u_{x_{j}} u_{x_{i} x_{j}} \\
& +a(|\nabla u|)^{2}\left|\nabla^{2} u\right|^{2}+a^{\prime}(|\nabla u|)^{2}(\nabla|\nabla u| \cdot \nabla u)^{2},
\end{aligned}
$$

where "." stands for scalar product in $\mathbb{R}^{n}$. After relabeling the indices, one has that

$$
\begin{aligned}
& a^{\prime}(|\nabla u|)^{2}(\nabla|\nabla u| \cdot \nabla u)^{2}+2 a(|\nabla u|) a^{\prime}(|\nabla u|) \sum_{i, j=1}^{n}|\nabla u|_{x_{i}} u_{x_{j}} u_{x_{i} x_{j}} \\
& +a(|\nabla u|)^{2}\left|\nabla^{2} u\right|^{2} \\
& =a(|\nabla u|)^{2}\left[\left(\frac{|\nabla u| a^{\prime}(|\nabla u|)}{a(|\nabla u|)}\right)^{2}\left(\sum_{i, k=1}^{n} \frac{u_{x_{k}} u_{x_{i}}}{|\nabla u|^{2}} u_{x_{k} x_{i}}\right)^{2}\right.
\end{aligned}
$$




$$
\left.+2 \sum_{i, j, k=1}^{n} \frac{|\nabla u| a^{\prime}(|\nabla u|)}{a(|\nabla u|)} \frac{u_{x_{k}} u_{x_{i}}}{|\nabla u|^{2}} u_{x_{k} x_{j}} u_{x_{i} x_{j}}+\sum_{i, j=1}^{n} u_{x_{i} x_{j}}^{2}\right] \text {. }
$$

Note that equation (3.3) holds at every point in $\Omega$ where $\nabla u \neq 0$, an assumption that we shall retain in the remaing part of the proof. Indeed, it is easily verified that if $\nabla u=0$, then inequality (3.1) holds with $C=1$.

Now, set

$$
\omega_{u}=\frac{\nabla u}{|\nabla u|}, \quad \vartheta_{u}=\frac{|\nabla u| a^{\prime}(|\nabla u|)}{a(|\nabla u|)}, \quad H_{u}=\nabla^{2} u .
$$

Observe that $\omega_{u} \in \mathbb{R}^{n}$, with $\left|\omega_{u}\right|=1, H_{u}$ is a symmetric matrix in $\mathbb{R}^{n \times n}$, and, by (2.2), $\vartheta_{u} \geqq i_{a}$. With this notation in place, the expression in square brackets on the right-hand side of (3.3) takes the form

$$
\vartheta_{u}^{2}\left(H_{u} \omega_{u} \cdot \omega_{u}\right)^{2}+2 \vartheta_{u} H_{u} \omega_{u} \cdot H_{u} \omega_{u}+\operatorname{tr}\left(H_{u}^{2}\right),
$$

where "tr" denotes the trace of a matrix. The proof of inequality (3.1) is thus reduced to showing that

$$
\vartheta_{u}^{2}\left(H_{u} \omega_{u} \cdot \omega_{u}\right)^{2}+2 \vartheta_{u} H_{u} \omega_{u} \cdot H_{u} \omega_{u}+\operatorname{tr}\left(H_{u}^{2}\right) \geqq C \operatorname{tr}\left(H_{u}^{2}\right)
$$

for some positive constant $C=C\left(n, i_{a}\right)$. To establish inequality (3.5), define the function $\psi: \mathbb{R} \times \mathbb{R}^{n} \times\left(\mathbb{R}^{n \times n} \backslash\{0\}\right) \rightarrow \mathbb{R}$ as

$$
\psi(\vartheta, \omega, H)=\vartheta^{2} \frac{(H \omega \cdot \omega)^{2}}{\operatorname{tr}\left(H^{2}\right)}+2 \vartheta \frac{H \omega \cdot H \omega}{\operatorname{tr}\left(H^{2}\right)}+1
$$

for $(\vartheta, \omega, H) \in \mathbb{R} \times \mathbb{R}^{n} \times\left(\mathbb{R}^{n \times n} \backslash\{0\}\right)$, and note that (3.5) will follow if we show that there exists a positive constant $C$ such that

$$
\psi(\vartheta, \omega, H) \geqq C
$$

if $\vartheta \geqq i_{a},|\omega|=1$ and $H$ is any non-vanishing symmetric matrix. For each fixed $\omega$ and $H$, the quadratic function $\vartheta \mapsto \psi(\vartheta, \omega, H)$ attains its minimum at $\vartheta=-\frac{H \omega \cdot H \omega}{(H \omega \cdot \omega)^{2}}$. We claim that

$$
-\frac{H \omega \cdot H \omega}{(H \omega \cdot \omega)^{2}} \leqq-1
$$

To verify equation (3.7), choose a basis in $\mathbb{R}^{n}$ in which $H$ has diagonal form $\operatorname{diag}\left(\lambda_{1}, \ldots \lambda_{n}\right)$, and let $\left(\omega_{1}, \ldots, \omega_{n}\right)$ denote the vector of the components of $\omega$ with respect to this basis. Then

$$
H \omega \cdot H \omega=\sum_{i=1}^{n} \lambda_{i}^{2} \omega_{i}^{2}, \quad H \omega \cdot \omega=\sum_{i=1}^{n} \lambda_{i} \omega_{i}^{2},
$$

and hence (3.7) follows, since

$$
\left(\sum_{i=1}^{n} \lambda_{i} \omega_{i}^{2}\right)^{2} \leqq\left(\sum_{i=1}^{n} \lambda_{i}^{2} \omega_{i}^{2}\right)\left(\sum_{i=1}^{n} \omega_{i}^{2}\right)=\left(\sum_{i=1}^{n} \lambda_{i}^{2} \omega_{i}^{2}\right),
$$


by Schwarz's inequality. Note that the equality holds in (3.8) inasmuch as $\sum_{i=1}^{n} \omega_{i}^{2}=1$. Owing to $(3.7), \psi(\vartheta, \omega, H)$ is a stricly increasing function of $\vartheta$ for $\vartheta \geqq-1$. Hence, by the first inequality in (2.2),

$$
\psi(\vartheta, \omega, H) \geqq \psi\left(i_{a}, \omega, H\right)>\psi(-1, \omega, H),
$$

if $\vartheta \geqq i_{a}$ and $|\omega|=1$. Assume, for a moment, that we know that

$$
\psi(-1, \omega, H) \geqq 0,
$$

if $|\omega|=1$ and $H$ is any symmetric matrix. Since $\psi$ is a continuous function, we deduce from (3.9) and (3.10) that

$$
\begin{aligned}
\psi(\vartheta, \omega, H) & \geqq \psi\left(i_{a}, \omega, H\right) \geqq \inf _{\left|\omega^{\prime}\right|=1, H^{\prime} \text { sym }} \psi\left(i_{a}, \omega^{\prime}, H^{\prime}\right) \\
& =\min _{\left|\omega^{\prime}\right|=1, H^{\prime} \text { sym },\left|H^{\prime}\right|=1} \psi\left(i_{a}, \omega^{\prime}, H^{\prime}\right)>0,
\end{aligned}
$$

if $|\omega|=1$ and $H$ is symmetric and different from 0. Hence (3.6) follows. Observe that the equality holds in (3.11) since $\psi$ is a homogenenous function of degree 0 in $H$.

It remains to prove inequality (3.10), namely that

$$
(H \omega \cdot \omega)^{2}-2 H \omega \cdot H \omega+\operatorname{tr}\left(H^{2}\right) \geqq 0,
$$

if $|\omega|=1$ and $H$ is symmetric.

After diagonalizing $H$ as above, inequality (3.12) reads

$$
\left(\sum_{i=1}^{n} \omega_{i}^{2} \lambda_{i}\right)^{2}-2 \sum_{i=1}^{n} \omega_{i}^{2} \lambda_{i}^{2}+\sum_{i=1}^{n} \lambda_{i}^{2} \geqq 0,
$$

if $\sum_{i=1}^{n} \omega_{i}^{2}=1$, and $\lambda_{i} \in \mathbb{R}, i=1, \ldots, n$. Inequality (3.13) is a consequence of the following lemma:

Lemma 3.2. Assume that $\eta_{i} \in \mathbb{R}$ are such that $\eta_{i} \geqq 0, i=1, \ldots n$, and $\sum_{i=1}^{n} \eta_{i} \leqq$ 1. Then

$$
\left(\sum_{i=1}^{n} \eta_{i} \lambda_{i}\right)^{2}-2 \sum_{i=1}^{n} \eta_{i} \lambda_{i}^{2}+\sum_{i=1}^{n} \lambda_{i}^{2} \geqq 0
$$

for every $\lambda_{i} \in \mathbb{R}, i=1, \ldots n$.

Proof. Given any numbers $\lambda_{i} \in \mathbb{R}, i=1, \ldots n$, define the function $\phi: \mathbb{R}^{n} \rightarrow \mathbb{R}$ by

$$
\phi(\eta)=\left(\sum_{i=1}^{n} \eta_{i} \lambda_{i}\right)^{2}-2 \sum_{i=1}^{n} \eta_{i} \lambda_{i}^{2}+\sum_{i=1}^{n} \lambda_{i}^{2}
$$

for $\eta=\left(\eta_{1}, \ldots, \eta_{n}\right) \in \mathbb{R}^{n}$, and the sets $G$ and $\Sigma$ as

$$
G=\left\{\eta \in \mathbb{R}^{n}: \eta_{i} \geqq 0, i=1, \ldots, n, \sum_{i=1}^{n} \eta_{i} \leqq 1\right\},
$$




$$
\Sigma=\left\{\eta \in \mathbb{R}^{n}: \eta_{i} \geqq 0, i=1, \ldots, n, \sum_{i=1}^{n} \eta_{i}=1\right\} .
$$

With these notations in place, inequality (3.14) reads

$$
\min _{\eta \in G} \phi(\eta) \geqq 0 .
$$

Observe that

$$
\min _{\eta \in G} \phi(\eta)=\min _{\eta \in \Sigma} \phi(\eta)
$$

Equation (3.16) is a consequence of the fact that, for every $\eta \in \Sigma$, the function $[0,1] \ni t \mapsto \phi(t \eta)$, a polynomial of degree 2 , is decreasing for $t \leqq \frac{\sum_{i=1}^{n} \eta_{i} \lambda_{i}^{2}}{\left(\sum_{i=1}^{n} \eta_{i} \lambda_{i}\right)^{2}}$, and the latter number is larger than or equal to 1 , since

$$
\left(\sum_{i=1}^{n} \eta_{i} \lambda_{i}\right)^{2} \leqq\left(\sum_{i=1}^{n} \eta_{i} \lambda_{i}^{2}\right)\left(\sum_{i=1}^{n} \eta_{i}\right)=\sum_{i=1}^{n} \eta_{i} \lambda_{i}^{2}
$$

if $\eta \in \Sigma$. Owing to (3.16), inequality (3.15) will follow if we show that

$$
\min _{\eta \in \Sigma} \phi(\eta) \geqq 0 .
$$

Inequality (3.17) can be proved by induction on $n$. If $n=1$, then it holds trivially. Assume now that $n \geqq 2$, and that (3.17) holds with $n$ replaced by $n-1$. Let $\bar{\eta}=\left(\bar{\eta}_{1}, \ldots, \bar{\eta}_{n}\right) \in \Sigma$ be such that

$$
\phi(\bar{\eta})=\min _{\eta \in \Sigma} \phi(\eta) .
$$

If $\bar{\eta}_{i}=0$ for some $i \in\{1, \ldots n\}$, then inequality (3.17) follows since we are assuming that it holds with $n$ replaced by $n-1$. Suppose next that $\bar{\eta}_{i} \neq 0$ for $i=1, \ldots, n$. The Lagrange multiplier condition then tells us that there exists $\mu \in \mathbb{R}$ such that

$$
\left\{\begin{array}{l}
\left(\sum_{i=1}^{n} \bar{\eta}_{i} \lambda_{i}-\lambda_{j}\right) \lambda_{j}+\mu=0, \text { for } j=1, \ldots, n . \\
\sum_{i=1}^{n} \bar{\eta}_{i}=1
\end{array}\right.
$$

Hence, in particular, on setting

$$
A=\lambda_{1}, \quad B=\sum_{i=1}^{n} \bar{\eta}_{i} \lambda_{i}-\lambda_{1}
$$

one has that

$$
\left(A-\lambda_{j}\right)\left(B-\lambda_{j}\right)=0 \text { for } j=1, \ldots, n .
$$

As a consequence of (3.19), for each $j=1, \ldots, n$,

$$
\text { either } \lambda_{j}=A \text { or } \lambda_{j}=B \text {. }
$$


Therefore,

$$
\lambda_{j}^{2}=(A+B) \lambda_{j}-A B
$$

for $j=1, \ldots, n$. Multiplying through this equation by $\eta_{j}$, for $j=1, \ldots, n$, adding the resulting equations, and making use of the second equation in (3.18) tell us that

$$
\sum_{j=1}^{n} \bar{\eta}_{j} \lambda_{j}^{2}=A^{2}+B^{2}+A B .
$$

Hence, we infer that

$$
\phi(\bar{\eta})=-A^{2}-B^{2}+\sum_{j=1}^{n} \lambda_{j}^{2} .
$$

Now, if $\lambda_{j}=A$ for every $j=1, \ldots, n$, then $B=0$, whence $\phi(\bar{\eta})=\sum_{j=2}^{n} \lambda_{j}^{2} \geqq 0$. If, instead, there exists $k>1$ such that $\lambda_{k}=B$, then $\phi(\bar{\eta})=-\lambda_{1}^{2}-\lambda_{k}^{2}+\sum_{j=1}^{n} \lambda_{j}^{2}=$ $\sum_{j \neq 1, k} \lambda_{j}^{2} \geqq 0$. Altogether, (3.17) follows.

\section{Global Estimates}

This section is devoted to proving Theorems 2.3 and 2.4. As a preliminary step, we briefly discuss the notion of generalized solutions adopted in our results, and recall some of their basic properties.

When the function $f$ on the right-hand side of the equation in problems (2.6) or (2.7) has a sufficiently high degree of summability to belong to the dual of the Sobolev type space associated with the function $a$, weak solutions to the relevant problems are well defined. In particular, the existence and uniqueness of these solutions can be established via standard monotonicity methods. We are not going to give details in this connection, since they are not needed for our purposes, and refer the interested reader to [23] for an account on this issue. We rather focus on the case when $f$ merely belongs to $L^{q}(\Omega)$ for any $q \geqq 1$. A definition of generalized solution in this case involves the use of spaces that consist of functions whose truncations are weakly differentiable. Specifically, given any $t>0$, let $T_{t}: \mathbb{R} \rightarrow \mathbb{R}$ denote the function defined as $T_{t}(s)=s$ if $|s| \leqq t$, and $T_{t}(s)=t \operatorname{sign}(s)$ if $|s|>t$. We set

$$
\mathcal{T}_{\text {loc }}^{1,1}(\Omega)=\left\{u \text { is measurable in } \Omega: T_{t}(u) \in W_{\text {loc }}^{1,1}(\Omega) \text { for every } t>0\right\} .
$$

The spaces $\mathcal{T}^{1,1}(\Omega)$ and $\mathcal{T}_{0}^{1,1}(\Omega)$ are defined accordingly, on replacing $W_{\text {loc }}^{1,1}(\Omega)$ with $W^{1,1}(\Omega)$ and $W_{0}^{1,1}(\Omega)$, respectively, on the right-hand side of (4.1). that

If $u \in \mathcal{T}_{\text {loc }}^{1,1}(\Omega)$, there exists a (unique) measurable function $Z_{u}: \Omega \rightarrow \mathbb{R}^{n}$ such

$$
\nabla\left(T_{t}(u)\right)=\chi_{\{|u|<t\}} Z_{u} \quad \text { a.e. in } \Omega
$$

for every $t>0-$ see [6, Lemma 2.1]. Here $\chi_{E}$ denotes the characteristic function of the set $E$. As already mentioned in Section 1, with abuse of notation, for every $u \in \mathcal{T}_{\text {loc }}^{1,1}(\Omega)$ we denote $Z_{u}$ simply by $\nabla u$. 
Assume that $f \in L^{q}(\Omega)$ for some $q \geqq 1$. A function $u \in \mathcal{T}_{0}^{1,1}(\Omega)$ will be called a generalized solution to the Dirichlet problem (2.6) if $a(|\nabla u|) \nabla u \in L^{1}(\Omega)$,

$$
\int_{\Omega} a(|\nabla u|) \nabla u \cdot \nabla \varphi \mathrm{d} x=\int_{\Omega} f \varphi \mathrm{d} x
$$

for every $\varphi \in C_{0}^{\infty}(\Omega)$, and there exists a sequence $\left\{f_{k}\right\} \subset C_{0}^{\infty}(\Omega)$ such that $f_{k} \rightarrow f$ in $L^{q}(\Omega)$ and the sequence of weak solutions $\left\{u_{k}\right\}$ to the problems (2.6) with $f$ replaced by $f_{k}$ satisfies

$$
u_{k} \rightarrow u \quad \text { a.e. in } \Omega \text {. }
$$

In (4.3), $\nabla u$ stands for the function $Z_{u}$ fulfilling (4.2).

By [23], there exists a unique generalized solution $u$ to problem (2.6), and

$$
\|a(|\nabla u|) \nabla u\|_{L^{1}(\Omega)} \leqq C\|f\|_{L^{1}(\Omega)}
$$

for some constant $C=C\left(|\Omega|, n, i_{a}, s_{a}\right)$. Moreover, if $\left\{f_{k}\right\}$ is any sequence as above, and $\left\{u_{k}\right\}$ is the associated sequence of weak solutions, then

$$
u_{k} \rightarrow u \text { and } \nabla u_{k} \rightarrow \nabla u \text { a.e. in } \Omega,
$$

up to subsequences.

The definition of generalized solutions to the Neumann problem (2.7) can be given analogously. Assume that $f \in L^{q}(\Omega)$ for some $q \geqq 1$, and satisfies (2.8). A function $u \in \mathcal{T}^{1,1}(\Omega)$ will be called a generalized solution to problem (2.7) if $a(|\nabla u|) \nabla u \in L^{1}(\Omega)$, equation (4.3) holds for every $\varphi \in C^{\infty}(\Omega) \cap W^{1, \infty}(\Omega)$, and there exists a sequence $\left\{f_{k}\right\} \subset C_{0}^{\infty}(\Omega)$, with $\int_{\Omega} f_{k}(x) \mathrm{d} x=0$ for $k \in \mathbb{N}$, such that $f_{k} \rightarrow f$ in $L^{q}(\Omega)$ and the sequence of (suitably normalized by additive constants) weak solutions $\left\{u_{k}\right\}$ to the problems (2.7) with $f$ replaced by $f_{k}$ satisfies

$$
u_{k} \rightarrow u \quad \text { a.e. in } \Omega \text {. }
$$

Owing to [23], if $\Omega$ is a bounded Lipschitz domain, then there exists a unique (up to addive constants) generalized solution $u$ to problem (2.7), and

$$
\|a(|\nabla u|) \nabla u\|_{L^{1}(\Omega)} \leqq C\|f\|_{L^{1}(\Omega)}
$$

for some constant $C=C\left(L_{\Omega}, d_{\Omega}, n, i_{a}, s_{a}\right)$. Moreover, if $\left\{f_{k}\right\}$ is any sequence as above, and $\left\{u_{k}\right\}$ is the associated sequence of (normalized) weak solutions, then

$$
u_{k} \rightarrow u \text { and } \nabla u_{k} \rightarrow \nabla u \text { a.e. in } \Omega \text {, }
$$

up to subsequences.

We conclude our background by recalling the definitions of Marcinkiewicz, and, more generally, Lorentz spaces that enter in our results. Let $(\mathcal{R}, m)$ be a $\sigma$-finite non atomic measure space. Given $q \in[1, \infty]$, the Marcinkiewicz space $L^{q, \infty}(\mathcal{R})$, also called weak $L^{q}(\mathcal{R})$ space, is the Banach function space endowed with the norm defined as

$$
\|\psi\|_{L^{q, \infty}(\mathcal{R})}=\sup _{s \in(0, m(\mathcal{R}))} s^{\frac{1}{q}} \psi^{* *}(s)
$$


for a measurable function $\psi$ on $\mathcal{R}$. Here, $\psi^{*}$ denotes the decreasing rearrangement of $\psi$, and $\psi^{* *}(s)=\frac{1}{s} \int_{0}^{s} \psi^{*}(r) \mathrm{d} r$ for $s>0$. The space $L^{q, \infty}(\mathcal{R})$ is borderline in the family of Lorentz spaces $L^{q, \sigma}(\mathcal{R})$, with $q \in[1, \infty]$ and $\sigma \in[1, \infty]$, that are equipped with the norm given by

$$
\|\psi\|_{L^{q, \sigma}(\mathcal{R})}=\left\|s^{\frac{1}{q}-\frac{1}{\sigma}} \psi^{* *}(s)\right\|_{L^{\sigma}(0, m(\mathcal{R}))}
$$

for $\psi$ as above. Indeed, one has that

$$
L^{q, \sigma_{1}}(\mathcal{R}) \varsubsetneqq L^{q, \sigma_{2}}(\mathcal{R}) \quad \text { if } q \in[1, \infty] \text { and } 1 \leqq \sigma_{1}<\sigma_{2} \leqq \infty .
$$

Also,

$$
L^{q, q}(\mathcal{R})=L^{q}(\mathcal{R}) \quad \text { for } q \in(1, \infty]
$$

up to equivalent norms. In the limiting case when $q=1$, the Marcinkiewicz type space $L^{1, \infty} \log L(\mathcal{R})$ comes into play in our results as a replacement for $L^{1, \infty}(\mathcal{R})$, which agrees with $L^{1}(\mathcal{R})$. A norm in $L^{1, \infty} \log L(\mathcal{R})$ is defined as

$$
\|\psi\|_{L^{1, \infty} \log L(\mathcal{R})}=\sup _{s \in(0, m(\mathcal{R}))} s \log \left(1+\frac{C}{s}\right) \psi^{* *}(s)
$$

for any fixed constant $C>m(\mathcal{R})$. Different constants $C$ result in equivalent norms in (4.11).

Proof of Theorem 2.4. We begin with a proof in the case when $u$ is the generalized solution to the Dirichlet problem (2.6). The needed variants for the solution to the Neumann problem (2.7) are indicated at the end.

The proof is split in steps. In Step 1 we establish the result under some additional regularity assumptions on $a, \Omega$ and $f$. The remaining steps are devoted to removing the extra assumptions, by approximation.

Step 1 Here, we assume that the following extra conditions are in force:

$$
\begin{aligned}
& f \in C_{0}^{\infty}(\Omega) ; \\
& \partial \Omega \in C^{\infty} ; \\
& a:[0, \infty) \rightarrow[0, \infty) \text { and } c_{1} \leqq a(t) \leqq c_{2} \text { for } t \geqq 0,
\end{aligned}
$$

for some constants $c_{2}>c_{1}>0$; the function $\mathcal{A}: \mathbb{R}^{n} \rightarrow[0, \infty)$, defined as $\mathcal{A}(\eta)=a(|\eta|)$ for $\eta \in \mathbb{R}^{n}$, is such that

$$
\mathcal{A} \in C^{\infty}\left(\mathbb{R}^{n}\right)
$$

Standard regularity results then ensure that the solution $u$ to problem (2.6) is classical, and $u \in C^{\infty}(\bar{\Omega})$ (see e.g. [21, Proof of Theorem 1.1] for details). Let $\xi \in C_{0}^{\infty}\left(\mathbb{R}^{n}\right)$. Squaring both sides of the equation in (2.6), multiplying through the resulting equation by $\xi^{2}$, integrating both sides over $\Omega$, and making use of inequality (3.1) yields 


$$
\begin{aligned}
\int_{\Omega} \xi^{2} f^{2} \mathrm{~d} x & =\int_{\Omega} \xi^{2}(\operatorname{div}(a(|\nabla u|) \nabla u))^{2} \mathrm{~d} x \\
\geqq & \int_{\Omega} \xi^{2}\left[\sum_{j=1}^{n}\left(a(|\nabla u|)^{2} u_{x_{j}} \Delta u\right)_{x_{j}}\right. \\
& \left.-\sum_{i=1}^{n}\left(a(|\nabla u|)^{2} \sum_{j=1}^{n} u_{x_{j}} u_{x_{i} x_{j}}\right)_{x_{i}}\right] \mathrm{d} x \\
& +C \int_{\Omega} \xi^{2} a(|\nabla u|)^{2}\left|\nabla^{2} u\right|^{2} \mathrm{~d} x
\end{aligned}
$$

for some constant $C=C\left(n, i_{a}\right)$. Now, [35, Equation $\left.(3,1,1,2)\right]$ tells us that

$$
\begin{aligned}
& \Delta u \frac{\partial u}{\partial v}-\sum_{i, j=1}^{n} u_{x_{i} x_{j}} u_{x_{i}} v_{j} \\
& =\operatorname{div}_{T}\left(\frac{\partial u}{\partial v} \nabla_{T} u\right)-\operatorname{tr} \mathcal{B}\left(\frac{\partial u}{\partial v}\right)^{2} \\
& \quad-\mathcal{B}\left(\nabla_{T} u, \nabla_{T} u\right)-2 \nabla_{T} u \cdot \nabla_{T} \frac{\partial u}{\partial v} \quad \text { on } \partial \Omega,
\end{aligned}
$$

where $\mathcal{B}$ is the second fundamental form on $\partial \Omega, \operatorname{tr} \mathcal{B}$ is its trace, $\operatorname{div}_{T}$ and $\nabla_{T}$ denote the divergence and the gradient operator on $\partial \Omega$, respectively, and $v_{j}$ stands for the $j$-th component of $v$. From the divergence theorem and equation (4.17) we deduce that

$$
\begin{aligned}
& \int_{\Omega} \xi^{2}\left[\sum_{j=1}^{n}\left(a(|\nabla u|)^{2} u_{x_{j}} \Delta u\right)_{x_{j}}-\sum_{i=1}^{n}\left(a(|\nabla u|)^{2} \sum_{j=1}^{n} u_{x_{j}} u_{x_{i} x_{j}}\right)_{x_{i}}\right] \mathrm{d} x \\
&=\int_{\partial \Omega} \xi^{2} a(|\nabla u|)^{2}\left[\Delta u \frac{\partial u}{\partial v}-\sum_{i, j=1}^{n} u_{x_{i} x_{j}} u_{x_{i}} v_{j}\right] d \mathcal{H}^{n-1}(x) \\
&-2 \int_{\Omega} a(|\nabla u|)^{2} \xi \nabla \xi \cdot\left[\Delta u \nabla u-\sum_{j=1}^{n} u_{x_{j}} \nabla u_{x_{j}}\right] \mathrm{d} x \\
&= \int_{\partial \Omega} \xi^{2} a(|\nabla u|)^{2}\left[\operatorname{div}_{T}\left(\frac{\partial u}{\partial v} \nabla_{T} u\right)-\operatorname{tr} \mathcal{B}\left(\frac{\partial u}{\partial v}\right)^{2}\right. \\
&\left.-\mathcal{B}\left(\nabla_{T} u, \nabla_{T} u\right)-2 \nabla_{T} u \cdot \nabla_{T} \frac{\partial u}{\partial v}\right] d \mathcal{H}^{n-1}(x) \\
&-2 \int_{\Omega} a(|\nabla u|)^{2} \xi \nabla \xi \cdot\left[\Delta u \nabla u-\sum_{j=1}^{n} u_{x_{j}} \nabla u_{x_{j}}\right] \mathrm{d} x .
\end{aligned}
$$

By Young's inequality, there exists a constant $C=C(n)$ such that 


$$
\begin{aligned}
& 2\left|\int_{\Omega} a(|\nabla u|)^{2} \xi \nabla \xi \cdot\left[\Delta u \nabla u-\sum_{j=1}^{n} u_{x_{j}} \nabla u_{x_{j}}\right] \mathrm{d} x\right| \\
& \quad \leqq \varepsilon C \int_{\Omega} \xi^{2} a(|\nabla u|)^{2}\left|\nabla^{2} u\right|^{2} \mathrm{~d} x+\frac{C}{\varepsilon} \int_{\Omega}|\nabla \xi|^{2} a(|\nabla u|)^{2}|\nabla u|^{2} \mathrm{~d} x
\end{aligned}
$$

for every $\varepsilon>0$. Equations (4.16), (4.18) and (4.19) ensure that there exist constants $C=C\left(n, i_{a}\right)$ and $C^{\prime}=C^{\prime}\left(n, i_{a}\right)$ such that

$$
\begin{aligned}
& C(1-\varepsilon) \int_{\Omega} \xi^{2} a(|\nabla u|)^{2}\left|\nabla^{2} u\right|^{2} \mathrm{~d} x \\
& \leqq \int_{\Omega} \xi^{2} f^{2} \mathrm{~d} x+\frac{C^{\prime}}{\varepsilon} \int_{\Omega}|\nabla \xi|^{2} a(|\nabla u|)^{2}|\nabla u|^{2} \mathrm{~d} x \\
& \quad+\mid \int_{\partial \Omega} \xi^{2} a(|\nabla u|)^{2}\left[\operatorname{div}_{T}\left(\frac{\partial u}{\partial v} \nabla_{T} u\right)-\operatorname{tr} \mathcal{B}\left(\frac{\partial u}{\partial v}\right)^{2}\right. \\
& \left.\quad-\mathcal{B}\left(\nabla_{T} u, \nabla_{T} u\right)-2 \nabla_{T} u \cdot \nabla_{T} \frac{\partial u}{\partial v}\right] d \mathcal{H}^{n-1}(x) \mid .
\end{aligned}
$$

On the other hand, owing to the Dirichlet boundary condition, $\nabla_{T} u=0$ on $\partial \Omega$, and hence

$$
\begin{aligned}
& \mid \int_{\partial \Omega} \xi^{2} a(|\nabla u|)^{2}\left[\operatorname{div}_{T}\left(\frac{\partial u}{\partial v} \nabla_{T} u\right)-\operatorname{tr} \mathcal{B}\left(\frac{\partial u}{\partial v}\right)^{2}\right. \\
&\left.-\mathcal{B}\left(\nabla_{T} u, \nabla_{T} u\right)-2 \nabla_{T} u \cdot \nabla_{T} \frac{\partial u}{\partial v}\right] d \mathcal{H}^{n-1}(x) \mid \\
&=\left|-\int_{\partial \Omega} \xi^{2} a(|\nabla u|)^{2} \operatorname{tr} \mathcal{B}\left(\frac{\partial u}{\partial v}\right)^{2} d \mathcal{H}^{n-1}(x)\right| \\
& \leqq C \int_{\partial \Omega} \xi^{2} a(|\nabla u|)^{2}|\nabla u|^{2}|\mathcal{B}| d \mathcal{H}^{n-1}(x)
\end{aligned}
$$

for some constant $C=C(n)$. Here, $|\mathcal{B}|$ denotes the norm of $\mathcal{B}$. Next, assume that

$$
\xi \in C_{0}^{\infty}\left(B_{r}\left(x_{0}\right)\right)
$$

for some $x_{0} \in \bar{\Omega}$ and $r>0$.

First, suppose that $x_{0} \in \partial \Omega$. Let us distinguish the cases when $n \geqq 3$ or $n=2$. When $n \geqq 3$, set

$$
Q(r)=\sup _{x \in \partial \Omega} \sup _{E \subset \partial \Omega \cap B_{r}(x)} \frac{\int_{E}|\mathcal{B}| d \mathcal{H}^{n-1}(y)}{\operatorname{cap}(E)} \quad \text { for } r>0,
$$

where $\operatorname{cap}(E)$ stands for the capacity of the set $E$ given by

$$
\operatorname{cap}(E)=\inf \left\{\int_{\mathbb{R}^{n}}|\nabla v|^{2} d y: v \in C_{0}^{1}\left(\mathbb{R}^{n}\right), v \geqq 1 \text { on } E\right\} .
$$


A weighted trace inequality on half-balls $[48,49]$ (see also [52, Section 2.5.2]), combined with a local flattening argument for $\Omega$ on a half-space, and with an evenextension argument from a half-space into $\mathbb{R}^{n}$, ensures that there exists a constant $C=C\left(L_{\Omega}, d_{\Omega}, n\right)$ such that

$$
\int_{\partial \Omega \cap B_{r}(x)} v^{2}|\mathcal{B}| d \mathcal{H}^{n-1}(y) \leqq C Q(r) \int_{\Omega \cap B_{r}(x)}|\nabla v|^{2} d y
$$

for every $x \in \partial \Omega, r>0$ and $v \in C_{0}^{1}\left(B_{r}(x)\right)$. Furthermore, a standard trace inequality tells us that that there exists a constant $C=C\left(L_{\Omega}, d_{\Omega}, n\right)$ such that

$$
\left(\int_{\partial \Omega \cap B_{r}(x)}|v|^{\frac{2(n-1)}{n-2}} d \mathcal{H}^{n-1}(y)\right)^{\frac{n-2}{n-1}} \leqq C \int_{\Omega \cap B_{r}(x)}|\nabla v|^{2} d y
$$

for every $x \in \partial \Omega, r>0$ and $v \in C_{0}^{1}\left(B_{r}(x)\right)$. By definition (4.24), choosing trial functions $v$ in (4.26) such that $v \geqq 1$ on $E$ implies that

$$
\mathcal{H}^{n-1}(E)^{\frac{n-2}{n-1}} \leqq C \operatorname{cap}(E)
$$

for every set $E \subset \partial \Omega$. By the Hardy-Littlewood inequality for the decreasing rearrangement (with respect to $\mathcal{H}^{n-1}$ ) [7, Chapter 2, Lemma 2.1], and (4.27),

$$
\begin{aligned}
Q(r) & \leqq \sup _{x \in \partial \Omega} \sup _{E \subset \partial \Omega \cap B_{r}(x)} \frac{\int_{0}^{\mathcal{H}^{n-1}(E)}\left(|\mathcal{B}|_{\mid \partial \Omega \cap B_{r}(x)}\right)^{*}(r) \mathrm{d} r}{\operatorname{cap}(E)} \\
& \leqq C \sup _{x \in \partial \Omega} \sup _{s>0} \frac{\int_{0}^{s}\left(|\mathcal{B}|_{\mid \partial \Omega \cap B_{r}(x)}\right)^{*}(r) \mathrm{d} r}{s^{\frac{n-2}{n-1}}}=C \sup _{x \in \partial \Omega}\|\mathcal{B}\|_{L^{n-1, \infty}\left(\partial \Omega \cap B_{r}(x)\right)}
\end{aligned}
$$

for some constant $C=C\left(L_{\Omega}, d_{\Omega}, n\right)$, for every $x \in \partial \Omega$ and $r>0$. An application of inequality (4.25) with $v=\xi a(|\nabla u|) u_{x_{i}}$, for $i=1, \ldots n$, yields, via (4.28),

$$
\begin{aligned}
& \int_{\partial \Omega} \xi^{2} a(|\nabla u|)^{2}|\nabla u|^{2}|\mathcal{B}| d \mathcal{H}^{n-1}(x) \\
& \leqq C \sup _{x \in \partial \Omega}\|\mathcal{B}\|_{L^{n-1, \infty}\left(\partial \Omega \cap B_{r}(x)\right)}\left(\int_{\Omega} \xi^{2} a(|\nabla u|)^{2}\left|\nabla^{2} u\right|^{2} \mathrm{~d} x\right. \\
& \left.\quad+\int_{\Omega}|\nabla \xi|^{2} a(|\nabla u|)^{2}|\nabla u|^{2} \mathrm{~d} x\right)
\end{aligned}
$$

for some constant $C=C\left(L_{\Omega}, d_{\Omega}, n, s_{a}\right)$. Note that here we have made use of the second inequality in (2.2) to infer that

$$
\left|\nabla\left(a(|\nabla u|) u_{x_{i}}\right)\right| \leqq C a(|\nabla u|)\left|\nabla^{2} u\right| \quad \text { in } \Omega,
$$

for $i=1, \ldots, n$, and for some constant $C=C\left(n, s_{a}\right)$. Combining equations (4.20) and (4.29) tells us that

$$
\left[C_{1}(1-\varepsilon)-C_{2} \sup _{x \in \partial \Omega}\|\mathcal{B}\|_{L^{n-1, \infty}\left(\partial \Omega \cap B_{r}(x)\right)}\right] \int_{\Omega} \xi^{2} a(|\nabla u|)^{2}\left|\nabla^{2} u\right|^{2} \mathrm{~d} x
$$




$$
\begin{aligned}
\leqq & \int_{\Omega} \xi^{2} f^{2} \mathrm{~d} x+\left[C_{2} \sup _{x \in \partial \Omega}\|\mathcal{B}\|_{L^{n-1, \infty}\left(\partial \Omega \cap B_{r}(x)\right)}\right. \\
& \left.+\frac{C_{3}}{\varepsilon}\right] \int_{\Omega}|\nabla \xi|^{2} a(|\nabla u|)^{2}|\nabla u|^{2} \mathrm{~d} x
\end{aligned}
$$

for some constants $C_{1}=C_{1}\left(n, i_{a}\right), C_{2}=C_{2}\left(L_{\Omega}, d_{\Omega}, n, s_{a}\right)$ and $C_{3}=C_{3}(n)$. If condition (2.11) is fulfilled with $c=\frac{C_{1}}{C_{2}}$, then there exists $r_{0}>0$ such that

$$
C_{1}(1-\varepsilon)-C_{2} \sup _{x \in \partial \Omega}\|\mathcal{B}\|_{L^{n-1, \infty}\left(\partial \Omega \cap B_{r}(x)\right)}>0
$$

if $0<r \leqq r_{0}$ and $\varepsilon$ is sufficiently small. Therefore, by inequality (4.31),

$$
\int_{\Omega} \xi^{2} a(|\nabla u|)^{2}\left|\nabla^{2} u\right|^{2} \mathrm{~d} x \leqq C \int_{\Omega} \xi^{2} f^{2} \mathrm{~d} x+C \int_{\Omega}|\nabla \xi|^{2} a(|\nabla u|)^{2}|\nabla u|^{2} \mathrm{~d} x
$$

for some constant $C=C\left(L_{\Omega}, d_{\Omega}, n, i_{a}, s_{a}\right)$, if $0<r \leqq r_{0}$ in (4.22).

In the case when $n=2$, define

$$
Q_{1}(r)=\sup _{x \in \partial \Omega} \sup _{E \subset \partial \Omega \cap B_{r}(x)} \frac{\int_{E}|\mathcal{B}| d \mathcal{H}^{1}(y)}{\operatorname{cap}_{B_{1}(x)}(E)} \quad \text { for } r \in(0,1),
$$

where $\operatorname{cap}_{B_{1}(x)}(E)$ stands for the capacity of the set $E$ given by

$$
\operatorname{cap}_{B_{1}(x)}(E)=\inf \left\{\int_{B_{1}(x)}|\nabla v|^{2} d y: v \in C_{0}^{1}\left(B_{1}(x)\right), v \geqq 1 \text { on } E\right\} .
$$

A counterpart of inequality (4.25) reads

$$
\int_{\partial \Omega \cap B_{r}(x)} v^{2}|\mathcal{B}| d \mathcal{H}^{1}(y) \leqq C Q_{1}(r) \int_{\Omega \cap B_{r}(x)}|\nabla v|^{2} d y
$$

for every $x \in \partial \Omega, r \in(0,1)$ and $v \in C_{0}^{1}\left(B_{r}(x)\right)$, where $C=C\left(L_{\Omega}, d_{\Omega}\right)$.

A borderline version of the trace inequality-see e.g. [1, Section 7.6.4]-ensures that there exists a constant $C=C\left(L_{\Omega}, d_{\Omega}, n\right)$ such that

$$
\sup _{E \subset \partial \Omega \cap B_{1}(x)} \frac{\left(\frac{1}{\mathcal{H}^{1}(E)} \int_{E} v d \mathcal{H}^{1}(y)\right)^{2}}{\log \left(1+\frac{\mathcal{H}^{1}\left(\partial \Omega \cap B_{1}(x)\right)}{\mathcal{H}^{1}(E)}\right)} \leqq C \int_{\Omega \cap B_{1}(x)}|\nabla v|^{2} d y
$$

for every $x \in \partial \Omega$, and $v \in C_{0}^{1}\left(B_{1}(x)\right)$. Notice that the left-hand side of (4.36) is equivalent to the square of the norm in an Orlicz space associated with the Young function $e^{t^{2}}-1$. The choice of trial functions $v$ in (4.36) such that $v \geqq 1$ on $E$ yields, via definition (4.34),

$$
\frac{1}{\log \left(1+\frac{C}{\mathcal{H}^{1}(E)}\right)} \leqq C \operatorname{cap}_{B_{1}(x)}(E)
$$


for some constant $C=C\left(L_{\Omega}, d_{\Omega}\right)$, and for every set $E \subset \partial \Omega \cap B_{1}(x)$. Thanks to (4.37) and to the Hardy-Littlewood inequality again,

$$
\begin{aligned}
Q_{1}(r) & \leqq \sup _{x \in \partial \Omega} \sup _{E \subset \partial \Omega \cap B_{r}(x)} \frac{\int_{0}^{\mathcal{H}^{1}(E)}\left(|\mathcal{B}|_{\left.\mid \partial \Omega \cap B_{r}(x)\right)^{*}(r) \mathrm{d} r}\right.}{\operatorname{cap}_{B_{1}(x)}(E)} \\
& \leqq C \sup _{x \in \partial \Omega} \sup _{s \in\left(0, \mathcal{H}^{1}\left(\partial \Omega \cap B_{r}(x)\right)\right)} \log \left(1+\frac{C}{s}\right) \int_{0}^{s}\left(|\mathcal{B}|_{\mid \partial \Omega \cap B_{r}(x)}\right)^{*}(r) \mathrm{d} r \\
& =C \sup _{x \in \partial \Omega}\|\mathcal{B}\|_{L^{1, \infty} \log L\left(\partial \Omega \cap B_{r}(x)\right)}
\end{aligned}
$$

for some constant $C=C\left(L_{\Omega}, d_{\Omega}\right)$, and for $r \in(0,1)$. Exploiting (4.38) instead of (4.28), and arguing as in the case when $n \geqq 3$, yields (4.32) for $n=2$.

When $x_{0} \in \Omega$ and $B_{r}\left(x_{0}\right) \subset \subset \Omega$, inequality (4.32) still holds, and its derivation is even simpler: it follows directly from (4.16), (4.18) and (4.19), since the boundary integral on the rightmost side of (4.18) vanishes in this case.

Now, let $\left\{B_{r_{k}}\right\}_{k \in K}$ be a finite covering of $\bar{\Omega}$ by balls $B_{r_{k}}$, with $r_{k} \leqq r_{0}$, such that either $B_{r_{k}}$ is centered on $\partial \Omega$, or $B_{r_{k}} \subset \subset \Omega$. Note that this covering can be chosen in such a way that the multiplicity of overlapping of the balls $B_{r_{k}}$ only depends on $n$. Let $\left\{\xi_{k}\right\}_{k \in K}$ be a family of functions such that $\xi_{k} \in C_{0}^{\infty}\left(B_{r_{k}}\right)$ and $\left\{\xi_{k}^{2}\right\}_{k \in K}$ is a partition of unity associated with the covering $\left\{B_{r_{k}}\right\}_{k \in K}$. Thus $\sum_{k \in K} \xi_{k}^{2}=1$ in $\bar{\Omega}$. On applying inequality (4.32) with $\xi=\xi_{k}$ for each $k$, and adding the resulting inequalities one obtains that

$$
\int_{\Omega} a(|\nabla u|)^{2}\left|\nabla^{2} u\right|^{2} \mathrm{~d} x \leqq C \int_{\Omega} f^{2} \mathrm{~d} x+C \int_{\Omega} a(|\nabla u|)^{2}|\nabla u|^{2} \mathrm{~d} x
$$

for some constant $C=C\left(L_{\Omega}, d_{\Omega}, n, i_{a}, s_{a}\right)$.

A version of the Sobolev inequalty entails that, for every $\sigma>0$, there exists a constant $C=C\left(L_{\Omega}, d_{\Omega}, n, \sigma\right)$ such that

$$
\int_{\Omega} v^{2} \mathrm{~d} x \leqq \sigma \int_{\Omega}|\nabla v|^{2} \mathrm{~d} x+C\left(\int_{\Omega}|v| \mathrm{d} x\right)^{2}
$$

for every $v \in W^{1,2}(\Omega)$ (see e.g. [52, Proof of Theorem 1.4.6/1]). Applying inequality (4.40) with $v=a(|\nabla u|) u_{x_{i}}, i=1, \ldots, n$, an recalling (4.30) tell us that

$$
\int_{\Omega} a(|\nabla u|)^{2}|\nabla u|^{2} \mathrm{~d} x \leqq \sigma C_{1} \int_{\Omega} a(|\nabla u|)^{2}\left|\nabla^{2} u\right|^{2} \mathrm{~d} x+C_{2}\left(\int_{\Omega} a(|\nabla u|)|\nabla u| \mathrm{d} x\right)^{2}
$$

for some constant $C_{1}=C_{1}\left(n, s_{a}\right)$ and $C_{2}=C_{2}\left(L_{\Omega}, d_{\Omega}, n, s_{a}, \sigma\right)$. On choosing $\sigma=\frac{1}{2 C C_{1}}$, where $C$ is the constant appearing in (4.39), and combining inequalities (4.39), (4.41) and (4.4) we conclude that

$$
\int_{\Omega} a(|\nabla u|)^{2}\left|\nabla^{2} u\right|^{2} \mathrm{~d} x \leqq C \int_{\Omega} f^{2} \mathrm{~d} x
$$


for some constant $C=C\left(L_{\Omega}, d_{\Omega}, n, i_{a}, s_{a}\right)$. Inequalities (4.41), (4.42) and (4.4) imply, via (4.30), that

$$
\|a(|\nabla u|) \nabla u\|_{W^{1,2}(\Omega)} \leqq C\|f\|_{L^{2}(\Omega)}
$$

for some constant $C=C\left(L_{\Omega}, d_{\Omega}, n, i_{a}, s_{a}\right)$. In particular, the dependence of the constant $C$ in (4.43) is in fact just through an upper bound for the quantities $L_{\Omega}, d_{\Omega}, s_{a}$, and a lower bound for $i_{a}$. This is crucial in view of the next steps.

Step 2 Here we remove assumptions (4.14) and (4.15). To this end, we make use of a family of functions $\left\{a_{\varepsilon}\right\}_{\varepsilon \in(0,1)}$, with $a_{\varepsilon}:[0, \infty) \rightarrow(0, \infty)$, given by

$$
a_{\varepsilon}(t)=\frac{\widetilde{a}_{\varepsilon}\left(\sqrt{\varepsilon+t^{2}}\right)+\varepsilon}{1+\varepsilon \widetilde{a}_{\varepsilon}\left(\sqrt{\varepsilon+t^{2}}\right)} \quad \text { for } t \geqq 0,
$$

where

$$
\widetilde{a}_{\varepsilon}(t)=\phi_{\varepsilon}(\log t) \quad \text { for } t>0
$$

and $\phi_{\varepsilon}: \mathbb{R} \rightarrow[0, \infty)$ is the convolution of a nonnegative smooth kernel $\varrho_{\varepsilon}$ such that $\int_{\mathbb{R}} \varrho_{\varepsilon}(t) \mathrm{d} t=1$ and $\operatorname{supp} \varrho_{\varepsilon} \subset(-\varepsilon, \varepsilon)$, with the function $\phi: \mathbb{R} \rightarrow[0, \infty)$ defined as

$$
\phi(s)=a\left(e^{s}\right) \quad \text { for } s \in \mathbb{R} .
$$

Combining [21, Lemma 3.3] and [22, Lemma 4.5] tells us that

$$
\begin{gathered}
a_{\varepsilon} \in C^{\infty}[0, \infty) \text { and } \varepsilon \leqq a_{\varepsilon}(t) \leqq \varepsilon^{-1} \text { for } t \geqq 0 ; \\
\min \left\{i_{a}, 0\right\} \leqq i_{a_{\varepsilon}} \leqq s_{a_{\varepsilon}} \leqq \max \left\{s_{a}, 0\right\}
\end{gathered}
$$

$\lim _{\varepsilon \rightarrow 0} a_{\varepsilon}(|\xi|) \xi=a(|\xi|) \xi \quad$ uniformly in $\left\{\xi \in \mathbb{R}^{n}:|\xi| \leqq M\right\}$ for every $M>0$

the function $\mathcal{A}_{\varepsilon}: \mathbb{R}^{n} \rightarrow[0, \infty)$, defined as $\mathcal{A}_{\varepsilon}(\eta)=a_{\varepsilon}(|\eta|)$ for $\eta \in \mathbb{R}^{n}$, is such that

$$
\mathcal{A}_{\varepsilon} \in C^{\infty}\left(\mathbb{R}^{n}\right) .
$$

Now, let $u_{\varepsilon}$ be the solution to the problem

$$
\begin{cases}-\operatorname{div}\left(a_{\varepsilon}\left(\left|\nabla u_{\varepsilon}\right|\right) \nabla u_{\varepsilon}\right)=f & \text { in } \Omega \\ u_{\varepsilon}=0 & \text { on } \partial \Omega .\end{cases}
$$

Owing to (4.44) and (4.47), the assumptions of Step 1 are fulfilled by problem (4.48). Thus, as a consequence of (4.43), there exists a constant $C=C\left(L_{\Omega}, d_{\Omega}, n, i_{a}, s_{a}\right)$ such that

$$
\left\|a_{\varepsilon}\left(\left|\nabla u_{\varepsilon}\right|\right) \nabla u_{\varepsilon}\right\|_{W^{1,2}(\Omega)} \leqq C\|f\|_{L^{2}(\Omega)}
$$


for $\varepsilon \in(0,1)$. Observe that the constant $C$ in (4.49) is actually independent of $\varepsilon$, thanks to (4.45). By (4.49), there exists a sequence $\left\{\varepsilon_{k}\right\}$ and a function $U: \Omega \rightarrow \mathbb{R}^{n}$ such that $U \in W^{1,2}(\Omega)$,

$$
a_{\varepsilon_{k}}\left(\left|\nabla u_{\varepsilon_{k}}\right|\right) \nabla u_{\varepsilon_{k}} \rightarrow U \quad \text { in } L^{2}(\Omega) \quad \text { and } \quad a_{\varepsilon_{k}}\left(\left|\nabla u_{\varepsilon_{k}}\right|\right) \nabla u_{\varepsilon_{k}} \rightarrow U \text { in } W^{1,2}(\Omega) \text {, }
$$

where the arrow " $\rightarrow$ " stands for weak convergence. On the other hand, a global estimate for $\left\|u_{\varepsilon_{k}}\right\|_{L^{\infty}(\Omega)}$ following from a result of [59], coupled with a local gradient estimate of [44, Theorem 1.7] ensures that $u_{\varepsilon_{k}} \in C_{\mathrm{loc}}^{1, \alpha}(\Omega)$, and that for any open set $\Omega^{\prime} \subset \subset \Omega$ there exists a constant $C$ such that

$$
\left\|u_{\varepsilon_{k}}\right\|_{C^{1, \alpha}\left(\Omega^{\prime}\right)} \leqq C
$$

for $k \in \mathbb{N}$. Thus, there exists a function $v \in C^{1}(\Omega)$ such that, on taking, if necessary, a subsequence,

$$
u_{\varepsilon_{k}} \rightarrow v \text { and } \nabla u_{\varepsilon_{k}} \rightarrow \nabla v \text { pointwise in } \Omega .
$$

In particular,

$$
a(|\nabla v|) \nabla v=U
$$

and hence

$$
a(|\nabla u|) \nabla u \in W^{1,2}(\Omega) .
$$

Testing the equation in (4.48) with any function $\varphi \in C_{0}^{\infty}(\Omega)$ yields

$$
\int_{\Omega} a_{\varepsilon_{k}}\left(\left|\nabla u_{\varepsilon_{k}}\right|\right) \nabla u_{\varepsilon_{k}} \cdot \nabla \varphi \mathrm{d} x=\int_{\Omega} f \varphi \mathrm{d} x .
$$

Owing to (4.50) and (4.53), on passing to the limit in (4.55) as $k \rightarrow \infty$ one deduces that

$$
\int_{\Omega} a(|\nabla v|) \nabla v \cdot \nabla \varphi \mathrm{d} x=\int_{\Omega} f \varphi \mathrm{d} x .
$$

By [21, Theorem 2.14], the sequence $\left\{u_{\varepsilon_{k}}\right\}$ is bounded in the Orlicz-Sobolev energy space associated with problem (4.48), and hence, by (4.46), the function $v$ belongs to the same space. This ensures that $a(|\nabla v|) \nabla v$ belongs to the dual of the Orlicz space in question. Thus, since, by (2.2), the space $C_{0}^{\infty}(\Omega)$ is dense in the relevant Orlicz-Sobolev space, equation (4.56) holds, in fact, for every function $\varphi$ in this space. Therefore, $v=u$, the weak solution to problem (2.6). Furthermore, by (4.49), we obtain via (4.50) and (4.53) that

$$
\|a(|\nabla u|) \nabla u\|_{W^{1,2}(\Omega)} \leqq C\|f\|_{L^{2}(\Omega)}
$$

for some constant $C=C\left(L_{\Omega}, d_{\Omega}, n, i_{a}, s_{a}\right)$.

Step 3 Here, we remove assumption (4.13). Via smooth approximation of the functions which locally describe $\partial \Omega$, one can construct a sequence $\left\{\Omega_{m}\right\}$ of open sets in $\mathbb{R}^{n}$ such that $\partial \Omega_{m} \in C^{\infty}, \Omega \subset \Omega_{m}, \lim _{m \rightarrow \infty}\left|\Omega_{m} \backslash \Omega\right|=0$, and the Hausdorff distance between $\Omega_{m}$ and $\Omega$ tends to 0 as $m \rightarrow \infty$. Also, there exists a constant $C=C(\Omega)$ such that

$$
L_{\Omega_{m}} \leqq C L_{\Omega} \text { and } d_{\Omega_{m}} \leqq C d_{\Omega}
$$


for $m \in \mathbb{N}$. Moreover, although smooth functions are neither dense in $W^{2} L^{n-1, \infty}$ if $n \geqq 3$, nor in $W^{2} L^{1, \infty} \log L$ if $n=2$, one has that

$$
\sup _{x \in \partial \Omega}\left\|\mathcal{B}_{m}\right\|_{L^{n-1, \infty}\left(\partial \Omega_{m} \cap B_{r}(x)\right)} \leqq C \sup _{x \in \partial \Omega}\|\mathcal{B}\|_{L^{n-1, \infty}\left(\partial \Omega \cap B_{r}(x)\right)} \quad \text { if } n \geqq 3,
$$

or

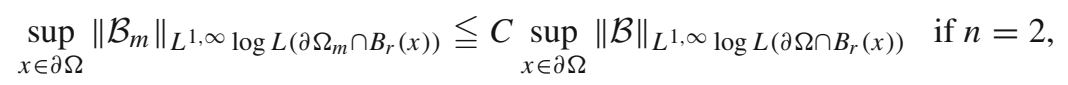

for some constant $C=C(\Omega)$, where $\mathcal{B}_{m}$ denotes the second fundamental form on $\partial \Omega_{m}$.

Let $u_{m}$ be the weak solution to the Dirichlet problem

$$
\begin{cases}-\operatorname{div}\left(a\left(\left|\nabla u_{m}\right|\right) \nabla u_{m}\right)=f & \text { in } \Omega_{m} \\ u_{m}=0 & \text { on } \partial \Omega_{m},\end{cases}
$$

where $f$ still fulfils (4.12), and is extended by 0 outside $\Omega$. By inequality (4.57) of Step 2,

$$
\left\|a\left(\left|\nabla u_{m}\right|\right) \nabla u_{m}\right\|_{W^{1,2}\left(\Omega_{m}\right)} \leqq C\|f\|_{L^{2}\left(\Omega_{m}\right)}=C\|f\|_{L^{2}(\Omega)},
$$

the constant $C$ being independent of $m$, by the properties of $\Omega_{m}$ mentioned above. Thanks to (4.60), the sequence $\left\{a\left(\left|\nabla u_{m}\right|\right) \nabla u_{m}\right\}$ is bounded in $W^{1,2}(\Omega)$, and hence there exists a subsequence, still denoted by $\left\{u_{m}\right\}$ and a function $U: \Omega \rightarrow \mathbb{R}^{n}$ such that $U \in W^{1,2}(\Omega)$,

$$
a\left(\left|\nabla u_{m}\right|\right) \nabla u_{m} \rightarrow U \quad \text { in } L^{2}(\Omega) \quad \text { and } \quad a\left(\left|\nabla u_{m}\right|\right) \nabla u_{m} \rightarrow U \text { in } W^{1,2}(\Omega) .
$$

By the local gradient estimate recalled in Step 2, there exists $\alpha \in(0,1)$ such that $u_{m} \in C_{\mathrm{loc}}^{1, \alpha}(\Omega)$, and for every open set $\Omega^{\prime} \subset \subset \Omega$ there exists a constant $C$, independent of $m$, such that

$$
\left\|u_{m}\right\|_{C^{1, \alpha}\left(\Omega^{\prime}\right)} \leqq C .
$$

Thus, on taking, if necessary, a further subsequence,

$$
u_{m} \rightarrow v \text { and } \nabla u_{m} \rightarrow \nabla v \text { pointwise in } \Omega,
$$

for some function $v \in C^{1}(\Omega)$. In particular,

$$
a\left(\left|\nabla u_{m}\right|\right) \nabla u_{m} \rightarrow a(|\nabla v|) \nabla v \text { pointwise in } \Omega .
$$

By (4.64) and (4.61),

$$
a(|\nabla v|) \nabla v=U \in W^{1,2}(\Omega) .
$$

Given any function $\varphi \in C_{0}^{\infty}(\Omega)$, on passing to the limit as $m \rightarrow \infty$ in the weak formulation of problem (4.59), namely in the equation

$$
\int_{\Omega_{m}} a\left(\left|\nabla u_{m}\right|\right) \nabla u_{m} \cdot \nabla \varphi \mathrm{d} x=\int_{\Omega_{m}} f \varphi \mathrm{d} x,
$$


we infer from (4.61) and (4.65) that

$$
\int_{\Omega} a(|\nabla v|) \nabla v \cdot \nabla \varphi \mathrm{d} x=\int_{\Omega} f \varphi \mathrm{d} x .
$$

An argument analogous to that exploited for equation (4.56) ensures that equation (4.67) continues to hold for every function $\varphi$ in the energy space associated with problem (2.6). Therefore, $u=v$, the weak solution to problem (2.6). Furthermore, owing to (4.60), (4.61) and (4.30),

$$
\|a(|\nabla u|) \nabla u\|_{W^{1,2}(\Omega)} \leqq C\|f\|_{L^{2}(\Omega)}
$$

for some constant $C=C\left(L_{\Omega}, d_{\Omega}, n, i_{a}, s_{a}\right)$.

Step 4 We conclude by removing the remaining additional assumption (4.12). Let $f \in L^{2}(\Omega)$. Owing to (4.5), given any sequence $\left\{f_{k}\right\} \subset C_{0}^{\infty}(\Omega)$ such that $f_{k} \rightarrow f$ in $L^{2}(\Omega)$, the sequence $\left\{u_{k}\right\}$ of the weak solutions to the Dirichlet problems

$$
\begin{cases}-\operatorname{div}\left(a\left(\left|\nabla u_{k}\right|\right) \nabla u_{k}\right)=f_{k} & \text { in } \Omega \\ u_{k}=0 & \text { on } \partial \Omega\end{cases}
$$

fullfils

$$
u_{k} \rightarrow u \text { and } \nabla u_{k} \rightarrow \nabla u \text { a.e. in } \Omega .
$$

By inequality (4.68) of the previous step, we have that $a\left(\left|\nabla u_{k}\right|\right) \nabla u_{k} \in W^{1,2}(\Omega)$, and there exist constants $C_{1}$ and $C_{2}$, depending on the same quantities as the constant $C$ in (4.68), such that

$$
\left\|a\left(\left|\nabla u_{k}\right|\right) \nabla u_{k}\right\|_{W^{1,2}(\Omega)} \leqq C_{1}\left\|f_{k}\right\|_{L^{2}(\Omega)} \leqq C_{2}\|f\|_{L^{2}(\Omega)} .
$$

Hence, the sequence $\left\{a\left(\left|\nabla u_{k}\right|\right) \nabla u_{k}\right\}$ is uniformly bounded in $W^{1,2}(\Omega)$, and there exists a subsequence, still indexed by $k$, and a function $U: \Omega \rightarrow \mathbb{R}^{n}$ such that $U \in W^{1,2}(\Omega)$ and

$$
a\left(\left|\nabla u_{k}\right|\right) \nabla u_{k} \rightarrow U \quad \text { in } L^{2}(\Omega) \quad \text { and } \quad a\left(\left|\nabla u_{k}\right|\right) \nabla u_{k} \rightarrow U \quad \text { in } W^{1,2}(\Omega) .
$$

From (4.70) we thus infer that $a(|\nabla u|) \nabla u=U \in W^{1,2}(\Omega)$, and the second inequality in (2.14) follows via (4.71) and (4.72). The first inequality is easily verified, via (4.30). The statement concerning the solution to the Dirichlet problem (2.6) is thus fully proved.

We point out hereafter the changes required for the solution to the Neumann problem (2.7).

Step 1 The additional assumption (2.8) has to be coupled with (4.12). Moreover, since $\frac{\partial u}{\partial v}=0$ on $\partial \Omega$, the middle term in the chain (4.21) is replaced with

$$
\left|-\int_{\partial \Omega} \xi^{2} a(|\nabla u|)^{2} \mathcal{B}\left(\nabla_{T} u, \nabla_{T} u\right) d \mathcal{H}^{n-1}(x)\right| .
$$

Step 2 The Dirichlet boundary condition in problem (4.48) must, of course, be replaced with the Neumann condition $\frac{\partial u_{\varepsilon}}{\partial v}=0$. The solution of the resulting Neumann problem is only unique up to additive constants. A bound of the form 
$\left\|u_{\varepsilon_{k}}-c_{k}\right\|_{L^{\infty}(\Omega)} \leqq C$ now holds for a suitable sequence $\left\{c_{k}\right\}$ with $c_{k} \in \mathbb{R}[18]$. Hence, $u_{\varepsilon_{k}}$ has to be replaced with $u_{\varepsilon_{k}}-c_{k}$ in equations (4.51) and (4.52). Moreover, the test functions $\varphi$ in equation (4.55) now belong to $W^{1, \infty}(\Omega)$.

Step 3 The Dirichlet problem (4.59) has to be replaced with the Neumann problem with boundary condition $\frac{\partial u_{m}}{\partial v}=0$. Accordingly, the corresponding sequence of solutions $\left\{u_{m}\right\}$ has to be normalized by a suitable sequence of additive constants. Passage to the limit as $m \rightarrow \infty$ in equation (4.66) can be justified as follows: extend any test function $\varphi \in W^{1, \infty}(\Omega)$ to a function in $W^{1, \infty}\left(\mathbb{R}^{n}\right)$, still denoted by $\varphi$; the left-hand side of equation (4.66) can be split as

$$
\begin{aligned}
\int_{\Omega_{m}} a\left(\left|\nabla u_{m}\right|\right) \nabla u_{m} \cdot \nabla \varphi \mathrm{d} x= & \int_{\Omega} a\left(\left|\nabla u_{m}\right|\right) \nabla u_{m} \cdot \nabla \varphi \mathrm{d} x \\
& +\int_{\Omega_{m} \backslash \Omega} a\left(\left|\nabla u_{m}\right|\right) \nabla u_{m} \cdot \nabla \varphi \mathrm{d} x,
\end{aligned}
$$

and the first integral on the right-hand side of (4.73) converges to

$$
\int_{\Omega} a(|\nabla v|) \nabla v \cdot \nabla \varphi \mathrm{d} x
$$

as $m \rightarrow \infty$, owing to (4.61) and (4.65). The second integral tends to 0 , by (4.60) and the fact that $\left|\Omega_{m} \backslash \Omega\right| \rightarrow 0$.

Step 4 The sequence of approximating functions $\left\{f_{k}\right\}$ has to fulfill the additional compatibility condition $\int_{\Omega} f_{k}(x) \mathrm{d} x=0$ for $k \in \mathbb{N}$. Moreover, the Dirichlet boundary condition in problem (4.69) has to be replaced with the Neumann condition $\frac{\partial u_{k}}{\partial v}=0$ on $\partial \Omega$.

Proof of Theorem 2.3. The proof parallels (and is even simpler than) that of Theorem 2.4. We limit ourselves to pointing out the variants and simplifications needed. Step 1 Assume that $\Omega, a$ and $f$ are as in Step 1 of the proof of Theorem 2.4 and that, in addition, $\Omega$ is convex. One can proceed as in that proof, and exploit the fact that the right-hand side of equation (4.17) is nonnegative owing to the convexity of $\Omega$, since it reduces to either

$$
-\operatorname{tr} \mathcal{B}\left(\frac{\partial u}{\partial v}\right)^{2} \geqq 0 \quad \text { or } \quad-\mathcal{B}\left(\nabla_{T} u, \nabla_{T} u\right) \geqq 0 \quad \text { on } \quad \partial \Omega,
$$

according to whether $u$ is the solution to the Dirichlet problem (2.6), or to the Neumann problem (2.7). Therefore, inequality (4.20) can be replaced with the stronger inequality

$C(1-\varepsilon) \int_{\Omega} \xi^{2} a(|\nabla u|)^{2}\left|\nabla^{2} u\right|^{2} \mathrm{~d} x \leqq \int_{\Omega} \xi^{2} f^{2} \mathrm{~d} x+\frac{C^{\prime}}{\varepsilon} \int_{\Omega}|\nabla \xi|^{2} a(|\nabla u|)^{2}|\nabla u|^{2} \mathrm{~d} x$.

Starting from this inequality, instead of (4.20), estimate (4.43) follows analogously. Step 2 The proof is the same as that of Theorem 2.4.

Step 3 The proof is analogous to that of Theorem 2.4, save that the approximating domains $\Omega_{m}$ have to be chosen in such a way that they are convex.

Step 4 The proof is the same as that of Theorem 2.4. 


\section{Local Estimates}

Here, we provide a proof of Theorem 2.1. The generalized local solutions to equation (2.1) considered in the statement can be defined as follows: assume that $f \in L_{\text {loc }}^{q}(\Omega)$ for some $q \geqq 1$; a function $u \in \mathcal{T}_{\text {loc }}^{1,1}(\Omega)$ is called a generalized local solution to equation (2.1) if $a(|\nabla u|) \nabla u \in L_{\text {loc }}^{1}(\Omega)$, equation (4.3) holds for every $\varphi \in C_{0}^{\infty}(\Omega)$, and there exists a sequence $\left\{f_{k}\right\} \subset C_{0}^{\infty}(\Omega)$ and a correpsonding sequence of local weak solutions $\left\{u_{k}\right\}$ to equation (2.1), with $f$ replaced by $f_{k}$, such that $f_{k} \rightarrow f$ in $L^{q}\left(\Omega^{\prime}\right)$,

$$
u_{k} \rightarrow u \text { and } \nabla u_{k} \rightarrow \nabla u \text { a.e. in } \Omega
$$

and

$$
\lim _{k \rightarrow \infty} \int_{\Omega^{\prime}} a\left(\left|\nabla u_{k}\right|\right)\left|\nabla u_{k}\right| \mathrm{d} x=\int_{\Omega^{\prime}} a(|\nabla u|)|\nabla u| \mathrm{d} x
$$

for every open set $\Omega^{\prime} \subset \subset \Omega$.

Note that, by the results from [23] recalled at the beginning of Section 4, the generalized solutions to the boundary value problems (2.6) and (2.7) are, in particular, generalized local solutions to equation (2.1).

Proof of Theorem 2.1. This proof follows the outline of that of Theorem 2.4. Some variants are however required, due to the local nature of the result. Of course, the step concerning the approximation of $\Omega$ by domains with a smooth boundary is not needed at all.

Step 1 Assume that the additional conditions (4.12) on $f$, and (4.14)-(4.15) on $a$ are in force, and let $u$ be a local weak solution to equation (2.1). Thanks to the current assumption on $a$ and $f$, the function $u$ is in fact a classical smooth solution. Let $B_{2 R}$ be any ball such that $B_{2 R} \subset \subset \Omega$, and let $R \leqq \sigma<\tau \leqq 2 R$. An application of inequality (4.20), with $\varepsilon=\frac{1}{2}$ and any function $\bar{\xi} \in C_{0}^{\infty}\left(\bar{B}_{\tau}\right)$ such that $\xi=1$ in $B_{\sigma}$ and $|\nabla \xi| \leqq C /(\tau-\sigma)$ for some constant $C=C(n)$, tells us that

$$
\int_{B_{\sigma}} a(|\nabla u|)^{2}\left|\nabla^{2} u\right|^{2} \mathrm{~d} x \leqq C \int_{B_{2 R}} f^{2} \mathrm{~d} x+\frac{C}{(\tau-\sigma)^{2}} \int_{B_{\tau} \backslash B_{\sigma}} a(|\nabla u|)^{2}|\nabla u|^{2} \mathrm{~d} x
$$

for some constant $C=C\left(n, i_{a}, s_{a}\right)$. We claim that there exists a constant $C=C(n)$ such that

$$
\int_{B_{\tau} \backslash B_{\sigma}} v^{2} \mathrm{~d} x \leqq C \delta^{2} R^{2} \int_{B_{\tau} \backslash B_{\sigma}}|\nabla v|^{2} \mathrm{~d} x+\frac{C}{\delta^{n}(\tau-\sigma) R^{n-1}}\left(\int_{B_{\tau} \backslash B_{\sigma}}|v| \mathrm{d} x\right)^{2}
$$

for every $\delta \in(0,1)$ and every $v \in W^{1,2}\left(B_{\tau} \backslash B_{\sigma}\right)$, provided that $R, \tau$ and $\sigma$ are as above. This claim can be verified as follows. Denote by $Q_{r}$ a cube of sidelength $r>0$. The inequality

$$
\int_{Q_{1}} v^{2} \mathrm{~d} x \leqq C_{1} \int_{Q_{1}}|\nabla v|^{2} \mathrm{~d} x+C_{2}\left(\int_{Q_{1}}|v| \mathrm{d} x\right)^{2}
$$


holds for every $v \in W^{1,2}\left(Q_{1}\right)$, for suitable constants $C_{1}=C_{1}(n)$ and $C_{2}(n)$. Given $\varepsilon \in(0,1)$, a scaling argument tells us that a parallel inequality holds in $Q_{\varepsilon}$, with $C_{1}$ replaced with $C_{1} \varepsilon^{2}$ and $C_{2}$ replaced with $C_{2} \varepsilon^{-n}$. A covering argument for $Q_{1}$ by cubes of sidelength $\varepsilon$ then yields inequality (5.5) with $C_{1}$ and $C_{2}$ replaced by $C_{1} \varepsilon^{2}$ and $C_{2} \varepsilon^{-n}$, respectively. Another scaling argument, applied to the resulting inequality in $Q_{1}$, provides us with the inequality

$$
\int_{Q_{\delta}} v^{2} \mathrm{~d} x \leqq C_{1}(\varepsilon \delta)^{2} \int_{Q_{\delta}}|\nabla v|^{2} \mathrm{~d} x+C_{2}(\varepsilon \delta)^{-n}\left(\int_{Q_{\delta}}|v| \mathrm{d} x\right)^{2}
$$

for every $v \in W^{1,2}\left(Q_{\delta}\right)$. Via a covering argument for $B_{2} \backslash B_{1}$ by (quasi)-cubes of suitable sidelength $\delta$, one infers from (5.6) that

$$
\int_{B_{2} \backslash B_{1}} v^{2} \mathrm{~d} x \leqq C \varepsilon^{2} \int_{B_{2} \backslash B_{1}}|\nabla v|^{2} \mathrm{~d} x+C \varepsilon^{-n}\left(\int_{B_{2} \backslash B_{1}}|v| \mathrm{d} x\right)^{2}
$$

for a suitable constant $C=C(n)$. Inequality (5.4) can be derived from (5.7) on mapping $B_{2} \backslash B_{1}$ into $B_{\tau} \backslash B_{\sigma}$ via the bijective map $\Phi: B_{2} \backslash B_{1} \rightarrow B_{\tau} \backslash B_{\sigma}$ defined as

$$
\Phi(x)=\frac{x}{|x|}[\sigma+(|x|-1)(\tau-\sigma)] \text { for } x \in B_{2} \backslash B_{1},
$$

and making use of the fact that

$$
c_{1}(\tau-\sigma) R^{n-1} \leqq|\operatorname{det}(\nabla \Phi(x))| \leqq c_{2}(\tau-\sigma) R^{n-1} \quad \text { for } x \in B_{2} \backslash B_{1}
$$

and

$$
|\nabla \Phi(x)| \leqq c_{2} R \text { for } x \in B_{2} \backslash B_{1},
$$

for suitable positive constants $c_{1}=c_{1}(n)$ and $c_{2}=c_{2}(n)$.

Choosing $\delta=(\tau-\sigma) / R$ in inequality (5.4), and applying the resulting inequality with $v=a(|\nabla u|) u_{x_{i}}$, for $i=1 \ldots, n$ yields

$$
\begin{aligned}
& \frac{1}{(\tau-\sigma)^{2}} \int_{B_{\tau} \backslash B_{\sigma}} a(|\nabla u|)^{2}|\nabla u|^{2} \mathrm{~d} x \\
& \leqq C \int_{B_{\tau} \backslash B_{\sigma}} a(|\nabla u|)^{2}\left|\nabla^{2} u\right|^{2} \mathrm{~d} x \\
& \quad+\frac{C R}{(\tau-\sigma)^{n+3}}\left(\int_{B_{\tau} \backslash B_{\sigma}} a(|\nabla u|)|\nabla u| \mathrm{d} x\right)^{2}
\end{aligned}
$$

for some constant $C=C\left(n, s_{a}\right)$. Observe that in (5.8) we have also made use of equation (4.30). Inequalities (5.3) and (5.8) imply that

$$
\begin{aligned}
& \int_{B_{\sigma}} a(|\nabla u|)^{2}\left|\nabla^{2} u\right|^{2} \mathrm{~d} x \leqq C \int_{B_{\tau} \backslash B_{\sigma}} a(|\nabla u|)^{2}\left|\nabla^{2} u\right|^{2} \mathrm{~d} x \\
& +C \int_{B_{2 R}} f^{2} \mathrm{~d} x+\frac{C R}{(\tau-\sigma)^{n+3}}\left(\int_{B_{2 R}} a(|\nabla u|)|\nabla u| \mathrm{d} x\right)^{2}
\end{aligned}
$$


for some constant $C=C\left(n, i_{a}, s_{a}\right)$. Adding the quantity $C \int_{B_{\sigma}} a(|\nabla u|)^{2}\left|\nabla^{2} u\right|^{2} \mathrm{~d} x$ to both sides of inequality (5.9), and dividing through the resulting inequality by $(1+C)$ enable us to deduce that

$$
\begin{aligned}
\int_{B_{\sigma}} a(|\nabla u|)^{2}\left|\nabla^{2} u\right|^{2} \mathrm{~d} x \leqq & \frac{C}{1+C} \int_{B_{\tau}} a(|\nabla u|)^{2}\left|\nabla^{2} u\right|^{2} \mathrm{~d} x \\
& +C^{\prime} \int_{B_{2 R}} f^{2} \mathrm{~d} x+\frac{C^{\prime} R}{(\tau-\sigma)^{n+3}}\left(\int_{B_{2 R}} a(|\nabla u|)|\nabla u| \mathrm{d} x\right)^{2}
\end{aligned}
$$

for positive constants $C=C\left(n, i_{a}, s_{a}\right)$ and $C^{\prime}=C^{\prime}\left(n, i_{a}, s_{a}\right)$. Inequality (5.10), via a standard iteration argument (see e.g. [34, Lemma 3.1, Chapter 5]), entails that

$$
\int_{B_{R}} a(|\nabla u|)^{2}\left|\nabla^{2} u\right|^{2} \mathrm{~d} x \leqq C \int_{B_{2 R}} f^{2} \mathrm{~d} x+\frac{C}{R^{n+2}}\left(\int_{B_{2 R}} a(|\nabla u|)|\nabla u| \mathrm{d} x\right)^{2}
$$

for some constant $C=C\left(n, i_{a}, s_{a}\right)$. On the other hand, a scaling argument applied to the Sobolev inequality (4.40), with $\Omega=B_{1}$ and $\sigma=1$, tells us that there exists a constant $C=C\left(n, s_{a}\right)$ such that

$$
\int_{B_{R}} a(|\nabla u|)^{2}|\nabla u|^{2} \mathrm{~d} x \leqq \int_{B_{R}} a(|\nabla u|)^{2}\left|\nabla^{2} u\right|^{2} \mathrm{~d} x+\frac{C}{R^{n}}\left(\int_{B_{R}} a(|\nabla u|)|\nabla u| \mathrm{d} x\right)^{2} .
$$

Coupling inequality (5.11) with (5.12) yields

$$
\|a(|\nabla u|) \nabla u\|_{W^{1,2}\left(B_{R}\right)} \leqq C\left(\|f\|_{L^{2}\left(B_{2 R}\right)}+\left(R^{-\frac{n}{2}}+R^{-\frac{n}{2}-1}\right)\|a(|\nabla u|) \nabla u\|_{L^{1}\left(B_{2 R}\right)}\right)
$$

for some constant $C=C\left(n, i_{a}, s_{a}\right)$.

Step 2 Assume that $u$ is a local solution to equation (2.1), with $a$ as in the statement, and $f$ still fulfilling (4.12). One has that $u \in L_{\text {loc }}^{\infty}(\Omega)$. This follows from [39, Theorem 5.1], or from gradient regularity results of [29]. As a consequence, by [44, Theorem 1.7], $u \in C_{\text {loc }}^{1, \alpha}(\Omega)$ for some $\alpha \in(0,1)$. Next, consider a family of functions $\left\{a_{\varepsilon}\right\}_{\varepsilon \in(0,1)}$ satisfying properties (4.44)-(4.47). Denote by $u_{\varepsilon}$ the solution to the problem

$$
\begin{cases}-\operatorname{div}\left(a_{\varepsilon}\left(\left|\nabla u_{\varepsilon}\right|\right) \nabla u_{\varepsilon}\right)=f & \text { in } B_{2 R} \\ u_{\varepsilon}=u & \text { on } \partial B_{2 R}\end{cases}
$$

Since $u \in C^{1, \alpha}\left(\overline{B_{2 R}}\right)$, by [44, Theorem 1.7 and subsequent remarks]

$$
\left\|u_{\varepsilon}\right\|_{C^{1, \beta}\left(\overline{\left.B_{2 R}\right)}\right.} \leqq C
$$

for some $\beta \in(0,1)$, and some constant $C$ independent of $\varepsilon$. Hence, in particular,

$$
\left\|a_{\varepsilon}\left(\left|\nabla u_{\varepsilon}\right|\right) \nabla u_{\varepsilon}\right\|_{L^{1}\left(B_{2 R}\right)} \leqq C
$$


for some constant independent of $\varepsilon$. The functions $a_{\varepsilon}$ satisfy the assumptions imposed on $a$ in Step 1. Thus, by inequality (5.13),

$$
\begin{aligned}
\left\|a_{\varepsilon}\left(\left|\nabla u_{\varepsilon}\right|\right) \nabla u_{\varepsilon}\right\|_{W^{1,2}\left(B_{R}\right)} \leqq & C\left(\|f\|_{L^{2}\left(B_{2 R}\right)}\right. \\
& \left.+\left(R^{-\frac{n}{2}}+R^{-\frac{n}{2}-1}\right)\left\|a_{\varepsilon}\left(\left|\nabla u_{\varepsilon}\right|\right) \nabla u_{\varepsilon}\right\|_{L^{1}\left(B_{2 R}\right)}\right),
\end{aligned}
$$

where, owing to (4.45), the constant $C=C\left(n, i_{a}, s_{a}\right)$, and, in particular, is indepedent of $\varepsilon$. Inequalities (5.16) and (5.17) ensure that the sequence $\left\{a_{\varepsilon}\left(\left|\nabla u_{\varepsilon}\right|\right) \nabla u_{\varepsilon}\right\}$ is bounded in $W^{1,2}\left(B_{R}\right)$, and hence there exists a function $U: B_{R} \rightarrow \mathbb{R}^{n}$, with $U \in W^{1,2}\left(B_{R}\right)$, and a sequence $\left\{\varepsilon_{k}\right\}$ such that

$$
a_{\varepsilon_{k}}\left(\left|\nabla u_{\varepsilon_{k}}\right|\right) \nabla u_{\varepsilon_{k}} \rightarrow U \text { in } L^{2}\left(B_{R}\right) \quad \text { and } \quad a_{\varepsilon_{k}}\left(\left|\nabla u_{\varepsilon_{k}}\right|\right) \nabla u_{\varepsilon_{k}} \rightarrow U \text { in } W^{1,2}\left(B_{R}\right) \text {. }
$$

Moreover, by (5.15), there exists a function $v \in C^{1}\left(\overline{B_{2 R}}\right)$ such that, up to subsequences,

$$
u_{\varepsilon_{k}} \rightarrow v \text { and } \nabla u_{\varepsilon_{k}} \rightarrow \nabla v
$$

uniformly in $\overline{B_{2 R}}$. In particular,

$$
v=u \text { on } \partial B_{2 R},
$$

inasmuch as $u_{\varepsilon_{k}}=u$ on $\partial B_{2 R}$ for every $k \in \mathbb{N}$. Thanks to (5.18) and (5.19),

$$
a(|\nabla v|) \nabla v=U \in W^{1,2}\left(B_{R}\right) .
$$

The definition of weak solution to problem (5.14) entails that

$$
\int_{B_{2 R}} a_{\varepsilon_{k}}\left(\left|\nabla u_{\varepsilon_{k}}\right|\right) \nabla u_{\varepsilon_{k}} \cdot \nabla \varphi \mathrm{d} x=\int_{B_{2 R}} f \varphi \mathrm{d} x
$$

for every $\varphi \in C_{0}^{\infty}\left(B_{2 R}\right)$. By (5.18) and (5.21), passing to the limit in (5.22) as $k \rightarrow \infty$ results in

$$
\int_{B_{2 R}} a(|\nabla v|) \nabla v \cdot \nabla \varphi \mathrm{d} x=\int_{B_{2 R}} f \varphi \mathrm{d} x .
$$

Since $v \in C^{1}\left(\overline{B_{2 R}}\right)$, equation (5.23) also holds for every $\varphi$ in the Orlicz-Sobolev energy space associated with the problem

$$
\begin{cases}-\operatorname{div}(a(|\nabla v|) \nabla v)=f & \text { in } B_{2 R} \\ v=u & \text { on } \partial B_{2 R} .\end{cases}
$$

Thus $v$ is the weak solution to this problem. Since $u$ solves the same problem, $u=v$ in $B_{2 R}$. Moreover, equations (5.17), (5.18), (5.19), (4.46) and (5.21) entail that $a(|\nabla u|) \nabla u \in W^{1,2}\left(B_{R}\right)$, and

$$
\|a(|\nabla u|) \nabla u\|_{W^{1,2}\left(B_{R}\right)} \leqq C\left(\|f\|_{L^{2}\left(B_{2 R}\right)}+\left(R^{-\frac{n}{2}}+R^{-\frac{n}{2}-1}\right)\|a(|\nabla u|) \nabla u\|_{L^{1}\left(B_{2 R}\right)}\right) .
$$

Step 3 Let $a$ and $f$ be as in the statement, let $u$ be a generalized local solution to equation (2.1), and let $f_{k}$ and $u_{k}$ be as in the definition of this kind of solution given 
at the begining of the present section. An application of Step 2 to $u_{k}$ tells us that $a\left(\left|\nabla u_{k}\right|\right) \nabla u_{k} \in W^{1,2}\left(B_{R}\right)$, and

$$
\begin{aligned}
\left\|a\left(\left|\nabla u_{k}\right|\right) \nabla u_{k}\right\|_{W^{1,2}\left(B_{R}\right)} \leqq & C\left(\left\|f_{k}\right\|_{L^{2}\left(B_{2 R}\right)}\right. \\
& \left.+\left(R^{-\frac{n}{2}}+R^{-\frac{n}{2}-1}\right)\left\|a\left(\left|\nabla u_{k}\right|\right) \nabla u_{k}\right\|_{L^{1}\left(B_{2 R}\right)}\right),
\end{aligned}
$$

where $C=C\left(n, i_{a}, s_{a}\right)$. Therefore, the sequence $\left\{a\left(\left|\nabla u_{k}\right|\right) \nabla u_{k}\right\}$ is bounded in $W^{1,2}\left(B_{R}\right)$, and hence there exists a function $U: B_{R} \rightarrow \mathbb{R}^{n}$, with $U \in W^{1,2}\left(B_{R}\right)$, and a subsequence, still indexed by $k$, such that

$$
a\left(\left|\nabla u_{k}\right|\right) \nabla u_{k} \rightarrow U \quad \text { in } L^{2}\left(B_{R}\right) \quad \text { and } \quad a\left(\left|\nabla u_{k}\right|\right) \nabla u_{k} \rightarrow U \quad \text { in } W^{1,2}\left(B_{R}\right) .
$$

By assumption (5.1), $\nabla u_{k} \rightarrow \nabla u$ a.e. in $\Omega$. Hence, owing to (5.27),

$$
a(|\nabla u|) \nabla u=U \quad \text { a.e. in } B_{R},
$$

and

$$
\liminf _{k \rightarrow \infty}\left\|a\left(\left|\nabla u_{k}\right|\right) \nabla u_{k}\right\|_{W^{1,2}\left(B_{R}\right)} \geqq\|a(|\nabla u|) \nabla u\|_{W^{1,2}\left(B_{R}\right)} .
$$

Inequality (2.5) follows from (5.26) and (5.29).

Acknowledgements. We wish to thank Connor Mooney for his valuable comments on a preliminary version of our paper. In particular, we are indebted to him for pointing out a flaw in the original formulation and proof of inequality (5.4), and for suggesting the present simplified proof of Lemma 3.2.

Funding. This research was partly funded by:

(i) Research Project of the Italian Ministry of University and Research (MIUR) Prin 2012 "Elliptic and parabolic partial differential equations: geometric aspects, related inequalities, and applications" (Grant Number 2012TC7588);

(ii) GNAMPA of the Italian INdAM - National Institute of High Mathematics (Grant Number not available);

(iii) Ministry of Education and Science of the Russian Federation (Grant Number 02.a03.21.0008).

\section{Compliance with Ethical Standards}

Conflict of interest. The authors declare that they have no conflict of interest.

Open Access This article is distributed under the terms of the Creative Commons Attribution 4.0 International License (http://creativecommons.org/licenses/by/4.0/), which permits unrestricted use, distribution, and reproduction in any medium, provided you give appropriate credit to the original author(s) and the source, provide a link to the Creative Commons license, and indicate if changes were made.

\section{References}

1. Adams, D.R., Hedberg, L.I.: Function Spaces and Potential Theory. Springer, Berlin, 1996 
2. Agmon, S., Douglis, A., Nirenberg, L.: Estimates near the boundary for solutions of elliptic partial differential equations satisfying general boundary conditions. I. Commun. Pure Appl. Math. 12, 623-727, 1959

3. Alvino, A., Cianchi, A., Maz'ya, V.G., Mercaldo, A.: Well-posed elliptic Neumann problems involving irregular data and domains. Ann. Inst. H. Poincaré Anal. Non Linéaire. 27, 1017-1054, 2010

4. Avelin, B., Kuusi, T., Mingione, G.: Nonlinear Calderón-Zygmund theory in a limiting case. Arch. Ration. Mech. Anal. 227, 663-714, 2018

5. BARONI, P.: Riesz potential estimates for a general class of quasilinear equations. Calc. Var. Partial Differ. Equ. 53, 803-846, 2015

6. Bénilan, P., Boccardo, L., Gallouët, T., Gariepy, R., Pierre, M., Vazquez, J.L.: An $L^{1}$-theory of existence and uniqueness of solutions of nonlinear elliptic equations. Ann. Sc. Norm. Sup. Pisa. 22, 241-273, 1995

7. Bennett, C., Sharpley, R.: Interpolation of Operators. Academic Press, Boston, 1988

8. BEIRÃo da VEIGA, H., CRISPo, F.: On the global $W^{2, q}$ regularity for nonlinear $N$-systems of the $p$-Laplacian type in $n$ space variables. Nonlinear Anal. 75, 4346-4354, 2012

9. Bernstein, S.: Sur la nature analytique des solutions des équations aux dérivées partielles du second ordre. Math. Ann. 59, 20-76, 1904

10. Boccardo, L., Gallouët, T.: Nonlinear elliptic and parabolic equations involving measure data. J. Funct. Anal. 87, 149-169, 1989

11. Breit, D., Cianchi, A., Diening, L., Kuusi, T., Schwarzacher, S.: Pointwise Calderón-Zygmund gradient estimates for the $p$-Laplace system. J. Math. Pures Appl. 114, 2018

12. Breit, D., Stroffolini, B., Verde, A.: A general regularity theorem for functionals with $\varphi$-growth. J. Math. Anal. Appl. 383, 226-233, 2011

13. Buliček, M., Diening, L., Schwarzacher, S.: Existence, uniqueness and optimal regularity results for very weak solutions to nonlinear elliptic systems. Anal. PDE 9, $1115-1151,2016$

14. Carozza, M., Kristensen, J., Passarelli di Napoli, A.: Higher differentiability of minimizers of convex variational integrals. Ann. Inst. H. Poincaré Anal. Non Linéaire. 28, 395-411, 2011

15. Cellina, A.: The regularity of solutions to some variational problems, including the $p$-Laplace equation, for $2 \leqq p<3$. ESAIM COCV 23, 1543-1553, 2017

16. Cellina, A.: The regularity of solutions to some variational problems, including the $p$-Laplace equation, for $3 \leqq p<4$, preprint

17. Chen, Y.Z., Di Benedetto, E.: Boundary estimates for solutions of nonlinear degenerate parabolic systems. J. Reine Angew. Math. 395, 102-131, 1989

18. Cianchi, A.: Elliptic equations on manifolds and isoperimetric inequalities. Proc. $R$. Soc. Edinburgh 114A, 213-227, 1990

19. Cianchi, A.: Boundednees of solutions to variational problems under general growth conditions. Commun. Partial Differ. Equ. 22, 1629-1646, 1997

20. Cianchi, A.: Local boundedness of minimizers of anisotropic functionals. Ann. Inst. H. Poincaré Anal. Non Linéaire 17, 147-168, 2000

21. Cianchi, A., Maz'ya, V.: Global Lipschitz regularity for a class of quasilinear elliptic equations. Commun. Partial Differ. Equ. 36, 100-133, 2011

22. Cianchi, A., Maz'Ya, V.: Global boundedness of the gradient for a class of nonlinear elliptic systems. Arch. Ration. Mech. Anal. 212, 129-177, 2014

23. Cianchi, A., MAZ'Ya, V.: Quasilinear elliptic problems with general growth and merely integrable, or measure, data. Nonlinear Anal. 164, 189-215, 2017

24. Crispo, F., Grisanti, C.R., Maremonti, P.: On the high regularity of solutions to the $p$-Laplacian boundary value problem in exterior domains. Ann. Math. Pure Appl. 195, 821-834, 2016

25. Dall'Aglio, A.: Approximated solutions of equations with $L^{1}$ data. Application to the $H$-convergence of quasi-linear parabolic equations. Ann. Math. Pure Appl. 170, 207-240, 1996 
26. Damascelli, L., Sciunzi, B.: Regularity, monotonicity and symmetry of positive solutions of $m$-Laplace equations. J. Differ. Equ. 206, 483-515, 2004

27. Di Benedetto, E.: $C^{1+\alpha}$ local regularity of weak solutions of degenerate elliptic equations. Nonlinear Anal. 7, 827-850, 1983

28. Di Benedetto, E., Manfredi, J.: On the higher integrability of the gradient of weak solutions of certain degenerate elliptic systems. Am. J. Math. 115, 1107-1134, 1993

29. Diening, L., KaplickÝ, P., SchwarZacher, S.: BMO estimates for the $p$-Laplacian. Nonlinear Anal. 75, 637-650, 2012

30. Diening, L., Stroffolini, B., Verde, A.: Everywhere regularity of functionals with $\phi$-growth. Manuscr. Math. 129, 449-481, 2009

31. Duzanr, F., Mingione, G.: Gradient estimates via non-linear potentials. Am. J. Math. 133, 1093-1149, 2011

32. Duzane, F., Mingione, G.: Gradient continuity estimates. Calc. Var. Partial Differ. Equ. 39, 379-418, 2010

33. Evans, L.C.: A new proof of local $C^{1, \alpha}$ regularity for solutions of certain degenerate elliptic P.D.E. J. Differ. Equ. 45, 356-373, 1982

34. GIAQuinta, M.: Multiple Integrals in the Calculus of Variations and Nonlinear Elliptic Systems, Annals of Mathematical Studies. Princeton University Press, Princeton, 1983

35. Grisvard, P.: Elliptic Problems in Nonsmooth Domains. Pitman, Boston, 1985

36. Hörmander, L.: Linear Partial Differential Operators. Springer, Berlin, 1963

37. IWANIEC, T.: Projections onto gradient fields and $L^{p}$-estimates for degenerated elliptic operators. Studia Math. 75, 293-312, 1983

38. Kilpeläinen, T., Malý, J.: The Wiener test and potential estimates for quasilinear elliptic equations. Acta Math. 172, 137-161, 1994

39. Korolev, A.G.: On boundedness of generalized solutions of elliptic differential equations with nonpower nonlinearities. MatH. Sb. 180, 78-100, 1989 (Russian)

40. Krol', I.N., MaZ'Ya, V.G.: On the absence of continuity and Hölder continuity of solutions of quasilinear elliptic equations near a nonregular boundary. Trudy Moskov. Mat. Oš̌č. 26, 1972 (Russian). English translation: Trans. Moscow Math. Soc. 26, $73-$ 93, 1972

41. Kuusi, T., Mingione, G.: Linear potentials in nonlinear potential theory. Arch. Ration. Mech. Anal. 207, 215-246, 2013

42. Ladyzenskaya, O.A., Ural'ceva, N.N.: Linear and quasilinear elliptic equations. Academic Press, New York, 1968

43. LewIS, J.L.: Regularity of the derivatives of solutions to certain degenerate elliptic equations. Indiana Univ. Math. J. 32, 849-858, 1983

44. Lieberman, G.M.: The natural generalization of the natural conditions of Ladyzenskaya and Ural'ceva for elliptic equations. Commun. Partial Differ. Equ. 16, 311-361, 1991

45. Lions, P.-L., Murat, F.: Sur les solutions renormalisées d'équations elliptiques non linéaires, manuscript

46. Lou, H.: On singular sets of local solutions to $p$-Laplace equations. Chin. Ann. Math. 29B, 521-530, 2008

47. Marcellini, P.: Regularity for elliptic equations with general growth conditions. $J$. Differ. Equ. 105, 296-333, 1993

48. MaZ'ya, V.: The negative spectrum of the higher-dimensional Schrödinger operator. Dokl. Akad. Nauk SSSR 144, 721-722, 1962 (Russian). English translation: Sov. Math. Dokl. 3, 1962

49. Mazya, V.: On the theory of the higher-dimensional Schrödinger operator. Izv. Akad. Nauk SSSR Ser. Mat. 28, 1145-1172, 1964 (Russian)

50. MaZ'YA, V.G.: Solvability in $W_{2}^{2}$ of the Dirichlet problem in a region with a smooth irregular boundary. Vestnik Leningrad. Univ. 22, 87-95, 1967 (Russian)

51. MaZ'YA, V.G.: On weak solutions of the Dirichlet and Neumann problems. Trusdy Moskov. Mat. Obšč. 20, 137-172, 1969 (Russian). English translation: Trans. Moscow Math. Soc. 20, 135-172, 1969 
52. MaZ'ya, V.G.: Sobolev Spaces with Applications to Elliptic Partial Differential Equations. Springer, Heidelberg, 2011

53. MAZ'ya, V.G., Shaposhnikova, T.O.: Theory of Sobolev Multipliers. With Applications to Differential and Integral Operators. Springer, Berlin, 2009

54. Mingione, G.: Gradient estimates below the duality exponent. Math. Ann. 346, 571627,2010

55. Murat, F.: Soluciones renormalizadas de EDP elṕticas no lineales, Preprint 93023, Laboratoire d'Analyse Numérique de l'Université Paris VI (1993)

56. Schauder, J.: Sur les équations linéaires du type elliptique a coefficients continuous. C. R. Acad. Sci. Paris 199, 1366-1368, 1934

57. Simon, J.: Régularité de solutions de problems nonlinéaires. C. R. Acad. Sci. Paris Sér. $A-B$ 282, A1351-A1354, 1976

58. Simon, L.: Interior gradient bounds for non-uniformly elliptic equations. Indiana Univ. Math. J. 25, 821-855, 1976

59. Talenti, G.: Nonlinear elliptic equations, rearrangements of functions and Orlicz spaces. Ann. Math. Pura Appl. 120, 159-184, 1979

60. TolKSDORF, P.: Regularity for a more general class of quasilinear elliptic equations. $J$. Differ. Equ. 51, 126-150, 1983

61. Uhlenbeck, K.: Regularity for a class of non-linear elliptic systems. Acta Math. 138, 219-240, 1977

62. Ural'Ceva, N.N.: Degenerate quasilinear elliptic systems. Zap. Naucn. Sem. Leningrad. Otdel. Mat. Inst. Steklov. (LOMI) 7, 184-222, 1968 (Russian)

\section{ANDREa Cianchi}

Dipartimento di Matematica e Informatica "U. Dini”,

Università di Firenze

Viale Morgagni 67/A,

50134 Florence, Italy.

e-mail: cianchi@unifi.it

and

VLADIMIR G. MAZ'Ya

Department of Mathematics,

Linköping University,

58183 Linköping,

Sweden.

and

VLADIMIR G. MAZ'YA

RUDN University

6 Miklukho-Maklay St,

Moscow, Russia 117198.

e-mail: vladimir.mazya@liu.se

(Received September 14, 2017 / Accepted February 2, 2018)

Published online May 2, 2018

(C) The Author(s) (2018) 